BNL-52285

UC-414 (High Energy Physics-

DOE/OSTI-4500-R75)

BNL --52285

DE91 010297

\title{
The Grating as an Accelerating Structure
}

\author{
R. C. Fernow
}

26 February, 1991

\author{
Physics Department \\ Brookhaven National Laboratory \\ Associated Unjersities, Inc. \\ Upton, Long Island, New York $1197 ?$ \\ Under Contract No. DE-AC02-76CH0001C , in the \\ UNITED STATES DEPARTMENT OF ENFRGY
}


ABSTRACT

This report considers the use of a diffraction grating as an accelerating structure for charged particle beams. We examine the functional dependence of the electromagnetic fields above the surface of a grating. Calculations are made of the strength of the accelerating modes for structures with $\pi$ and $2 \pi$ phase advance per period and for incident waves polarized with either the $E$ or $H$ vector along the grooves of the grating. We consider examples of using gratings in a laser linac and in a grating lens, we also briefly examine previous results published about this subiect. 


\section{CONTENTS}

1. Introduction 1

2. General form of the diffracted field 2

3. Synchronism condition 6

4. Traveling wave field components 10

5. Shallow sinusoidal grating 11

$\begin{array}{lr}\text { 6. Standing wave fields } & 18\end{array}$

7. Forces 21

8. Pi mode accelerating structures 24

9. Vee grooved structures 28

10. Laser linac 33

11. Grating lens 33

12. Survey of the theoretical literature 36

13. Survey of the experimental literature 38

14. Conclusions 40

Endnotes and references 41 


\section{Introduction}

The development of accelerators has progressed steadily throughout the twentieth century. The so-called "Livingston plot" shows[1] that the highest available accelerator energy has grown by more than an order of magnitude per decade in the period from 1930 to 1980. Accelerator physicists have accomplished this remarkable achievement both by continually optimizing existing techniques and by searching for new ones. As part of this effort a number of people have proposed using the enormous peak power available from lasers as a power source for an accelerator. Conventional diskloaded accelerator structures would not be useable with lasers because of the very short wavelengths involved. This has lead in turn to the investigation of a number of alternative schemes for coupling the radiation field to the particle beam. One of these ideas can be thought of as taking a disk-loaded cylindrical cavity, cutting it longitudinally along one edge, and then spreading it out flat. The resulting structure is a grating. Because it is open to free space on one side the grating has the advantage that it can be scaled to any desired wavelength and still couple to the radiation from the power source.

The first part of this report is concerned with the form of the electromagnetic fields that can exist above a grating surface. We discuss the requirements for phase synchronization between the radiation field and the particle beam. We look at specific solutions of the field equations for the case of a shallow sinusoidal grating boundary. We discuss the optimization of the shape of the boundary surface to obtain the maximum accelerating field enhancement. We consider the forces on a particle above the grating and give examples of a grating accelerator and a grating lens. The second part of the report consists of a trief review of the 1 iterature on theoretical and experimental work using gratings as accelerator structures.

We will use the coordinate system shown in Fig. 1, where the particle travels along the $z$ axis and free space is in the $y$ direction above the grating surface. We confine our discussions in this report to true gratings, which we define specifically as

(1) a perfectly conducting structure

(2) uniform along the $x$ direction

(3) periodic along the $z$ direction

(4) with free space in the region $y>0$. 


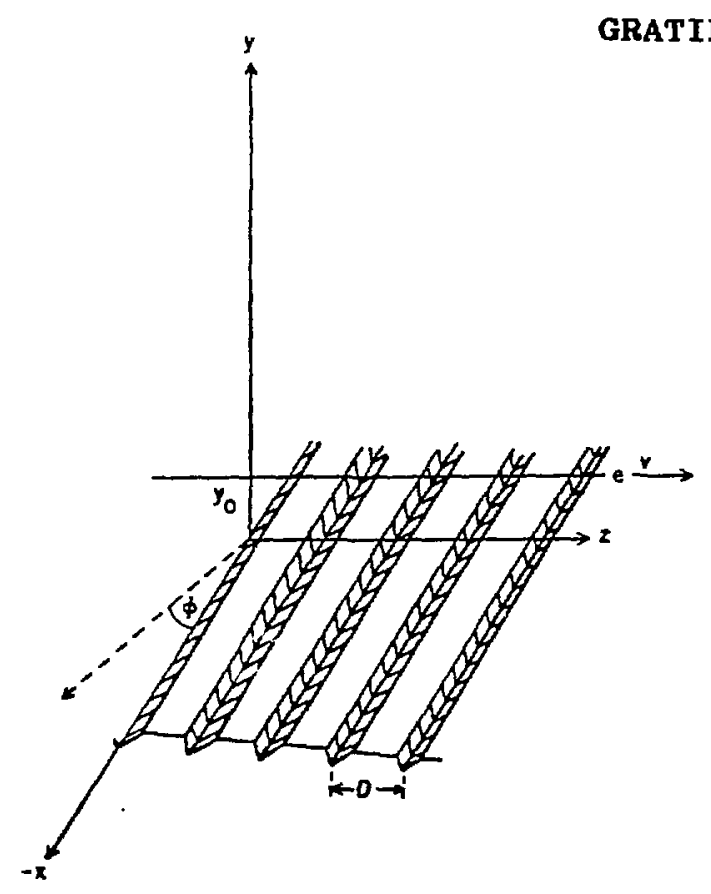

Fig. 1 Grating coordinate system. $x$ :along groove, $y$ : normal to mean surface, $z$ :along beam direction, $y_{0}$ :mean beam height, D:grating period, $\phi$ :angle in $x-y$ plane.

\section{General form of the diffracted field}

It is a well known result from the electromagnetic theory of gratings that Maxwell's equations can be broken into two independent sets of uncoupled equations[2,p.6]. The solutions to the first set of equations has $E_{*} * 0$ in general and $H_{x}=0$ everywhere. We refer to this as the E-polarization case. The total value for $E_{d}$ must satisfy the boundary condition $E_{x}=0$ on the grating surface. The solutions to the second class of equations has $H_{x} * 0$ in general and $E_{x}=0$ everywhere. We will refer to this as the H-polarization case. The total value of $H_{z}$ has to satisfy the boundary condition $\partial_{\mathrm{z}} / \partial \mathrm{n}=0$ on the grating surface, where $n$ is the outwardly directed normal vector at the point on the surface. As we shall show later, all the other components of $\underline{E}$ and $\underline{H}$ can be calculated from $E_{z}$ and $H_{x}$. This situation is similar to that in waveguide theory where all the transverse components of the field can be calculated from the longitudinal components. 


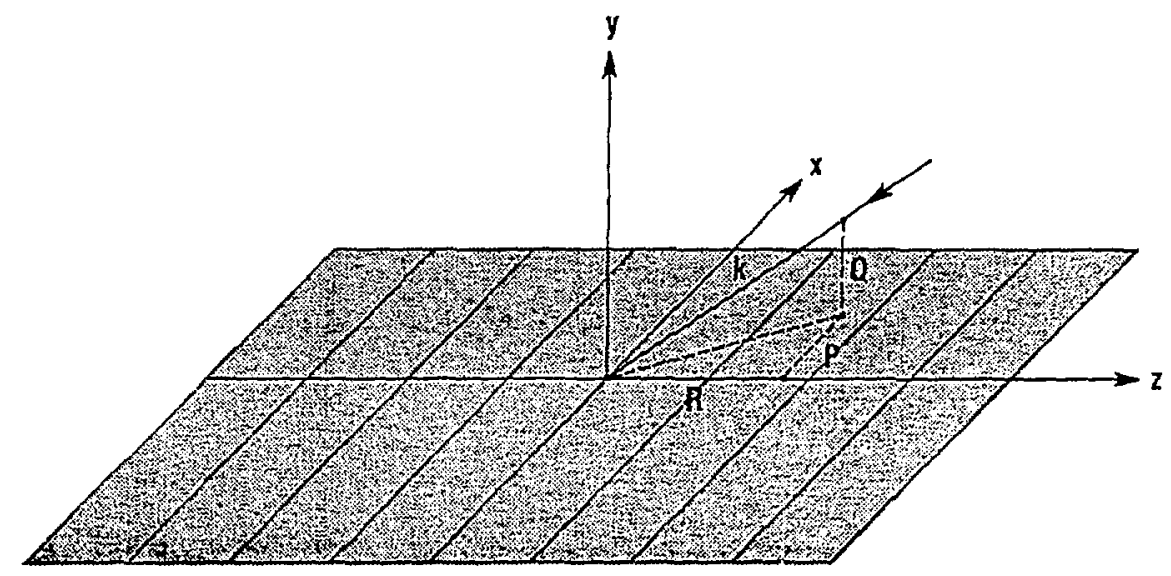

Fig. 2 Components of the incident wavevector. $k$ :magnitude, $P$ :component along $x, Q$ : component along $y$, $F$ : component along $z$.

We now want to consider the general functional form of the these solutions. To begin we assume that all the field components have an $\exp \{-i \omega t\}$ time dependence. Assume that a plane wave is incident on the grating surface as shown in Fig. 2. Let $F$ represent either of the fundamental field components $E_{3}$ or $H_{2}$. The corresponding component of the incident wave can be written

$$
F^{i n}=F_{0} e^{i P x} e^{-i \phi y} e^{i R x}
$$

where we use capital letters to refer to the components of the wavevector of the incoming wave. Every component of the field must satisfy the Helmholtz equation

$$
\left(\nabla^{2}+k^{2}\right) F=0
$$

Applying this to the incident wave gives the relation

$$
k^{2}=\frac{\omega^{2}}{C^{2}}=P^{2}+Q^{2}+R^{2}
$$

where $\theta$ is the frequency of the incident wave and $c$ is the velocity of light in vacuum. 
After the incident wave reaches the grating surface there must also exist a diffracted field, which we designate as $F^{\text {. The total }}$ electromagnetic field, consisting of the incident and diffracted fields, must satisfy the boundary conditions at the grating surface. The diffracted field can include the reflected wave and other outgoing radiation. In addition it can include a collection of evanescent surface waves, whose amplitude decays exponentially as one moves vertically away from the grating surface.

The boundary conditions give an intimate relation between the diffracted field and the incident field on the grating surface. In particular, the conditions must be satisfied for any value of $x$. Then, since the structure is uniform in the $x$ direction and cannot therefore introduce any additional $x$ dependence, we can only satisfy the conditions if the diffracted field has the same $x$ dependence as the incident field. Therefore, we know that the diffracted field can be written

$$
F^{D}(x, y, z)=h(y, z) e^{i P x}
$$

where $h(y, z)$ is a function that remains to be determined.

Now consider the effect of the periodicity along $z$. First remove

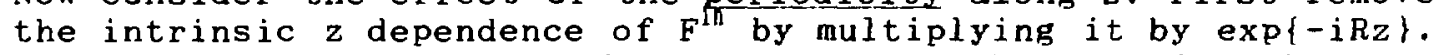
Since the boundary is periodic along $z$, this function has the same value at $z_{0}+D$ that it does at $z_{0}$. Therefore, the function can be written as the Fourier series in $z$

$$
F^{i n} e^{-i R z}=\sum_{n} g_{n}^{\prime}(y) e^{i \frac{2 \times n z}{D}}
$$
Because of the $E_{I}$ boundary condition we can rewrite this in terms
of $F$

$$
-F^{D} \theta^{-1 R z}=\sum_{n} g_{a}^{\prime}(y) e^{1 \frac{2 \pi n z}{D}}
$$

Therefore, if we absorb the minus sign in the definition of the function $g$, we can write the diffracted field as

$$
F^{D}=\sum_{D} e^{1 P x} g_{D}(y) e^{i x_{n} x}
$$

where we define

$$
I_{n}=R+\frac{2 \pi n}{D}
$$

A similar argument holds for the $H_{y}$ boundary condition. Each of the 
terms in the sum is referred to as a space harmonic.

Finally, if we restrict ourselves to $y$ values above the highest point of the grating surface, we know that $F^{b}$ has to satisfy the Helmholtz equation for any value of $z$. Interchanging the derivatives and the summation, we find that

$$
\sum_{i}\left(-P^{2}+\partial_{y}^{2}-I_{z}^{2}+k^{2}\right) e^{1 P x} g_{n}(y) e^{1 I_{0} x}=0
$$

Since the $x$ dependence is constant, we can cancel it and rewrite this as

$$
\sum_{n}\left[\left(\partial_{y}^{2}+\left(k^{2}-P^{2}-r_{n}^{2}\right)\right) g_{D}(y)\right] e^{1 I_{n} 2}=0
$$

This equation basically expresses the constant 0 as a complex Fourier series. Therefore, the coefficient of every term must be 0 . This gives the differential equation for $g_{n}(y)$

$$
\left[\partial_{y}^{2}+\left(k^{2}-P^{2}-r_{n}^{2}\right)\right] g_{n}(y)=0
$$

The equation has the solution

$$
g_{n}(y)=f_{n} e^{i q_{n} y}
$$

where $f_{n}$ is a constant and

$$
g_{n}=\sqrt{k^{2}-P^{2}-r_{2}^{2}}
$$

The constants $f_{n}$ give the coupling strengths into the various space harmonics. They depend on the angles of incidence and polarization of the incident wave and on the detailed shape of the grating surface. Note that the wavevector $q_{n}$ can be real or complex. The solutions with real $q_{n}$ correspond to outgoing radiation, which if they exist are important as a source of energy loss. Thus the diffracted field is given by

$$
F^{D}=\sum_{z} f_{x} e^{i P_{x}} e^{i \nabla_{a} y} e^{i r_{n} z}
$$

This is known as the Ravleigh expansion since it was first derived[3] by Lord Rayleigh in 1907 .

It is important to note that the above proof leading to this expansion is only rigorously valid in the space above the maximun of the grating profile[2,p.15]. In the space between the grooves of the grating the diffracted field may no longer have the simple dependence $\exp \left\{\mathrm{ir}_{n} z\right\}$ for any value of $z$ because certain values of 
$z$ now correspond to the grating boundary. The assumption that the Rayleigh expansion is valid everywhere above the grating including the region between the grooves is known as the Rayleigh assumption. It can be proven[2,p.61] to be false for E-polarized light incident on sinusoidal gratings of period $D$ and amplitude $h$ when $2 \pi h / D>$ 0.448 . However, the Rayleigh assumption can lead to accurate results for shallow gratings[2,p.18]. Rigorous solutions for the diffracted field anywhere above a grating with arbitrary surface profile can be written in terms of integral equations over the grating boundary $[2,4]$.

We note that the $n=0$ space harmonic has a special meaning. In this case $r_{n}=R$ and $q_{n}=+Q$. This corresponds to a real outgoing wave representing the reflected incident wave.

For acceleration we are interested in solutions for the diffracted field that travel along and above the maximum height of the grating surface. Thus the Rayleigh expansion will be valid for the field components. We require solutions that are bound to the gratine surface in order for the particles to be continuously accelerated by interaction with the field. Thus the accelerating mode must have the evanescent form

$$
f_{n} e^{i P x} e^{-g_{n} y} e^{i x_{n} z}
$$

and the wavevectors must satisfy the inequality

$$
p^{2}+r_{n}^{2}>k^{2}
$$

\section{Synchronism condition}

Now consider a charged particle crossing the grating. The particle travels along the $z$ axis by definition. The various space harmonics have phase velocities along $z$ given by

$$
v_{p 0}=\frac{c k}{R+\frac{2 \pi n}{D}}
$$

Note that for any given value of $R$ it is possible to choose a 
positive value of $n$ large enough to make $v_{p}<c$. The condition for continuous acceleration is that the particle velocity must match the phase velocity of the electromagnetic wave along the $z$ axis.

$$
\beta c=v_{p}=\frac{\omega}{I_{n}}=\frac{c k}{r_{n}}
$$

Thus the synchronism condition is

$$
\beta=\frac{k}{r_{\mathrm{n}}}
$$

Let us consider a solution for the diffracted field that does not depend on $x(i . e . P=0)$. The Helmholtz equation gives

$$
q_{n}^{2}-r_{n}^{2}+k^{2}=0
$$

Substituting for $r_{n}$ in the synchronism condition we find

$$
\beta=\frac{k}{\sqrt{q_{n}^{2}+k^{2}}}
$$

However, as the particle velocity becomes relativistic and $B \rightarrow 1$, we must have $q_{n} \rightarrow 0$ and $r_{n} \rightarrow k$. This corresponds to a plane wave travelling along the $z$ axis. Then because it is transversely polarized there will not be any component of electric field to accelerate the particle. This conclusion is sometimes referred to as Lawson's Theorem for Gratings: the field configuration above a grating must have a variation along the groove $(x)$ direction in order to support relativistic accelerating modes[5]. As a consequence of this we cannot excite a relativistic accelerating mode by bringing the incident radiation onto the grating from any direction in the $y-z$ plane and in particular by bringing the radiation directly down normal to the surface.

Now consider the case where the incident wave is not in the $y-z$ plane (i.e. $P \neq 0$ ). Now the Helmholcz equation gives

$$
-P^{2}+q_{n}^{2}-r_{n}^{2}+k^{2}=0
$$

and the synchronism condition is

$$
\beta=\frac{k}{\sqrt{k^{2}-P^{2}+q_{a}^{2}}}
$$

Note that relativistic synchronization is now possible, provided that $q_{n}=P$. The fact that a surface wave traveling at an angle to the particle direction could provide relativistic acceleration was 
first noted by Palmer[5].

Now let us rewrite the synchronism condition in terms of the space harmonics.

$$
k=\beta\left(R+\frac{2 \pi n}{D}\right)
$$

From Fig. 3 we see that $R=k \cos (\theta)$, where $\theta$ is whe space angle between the incident wave and the particle (z) axis.

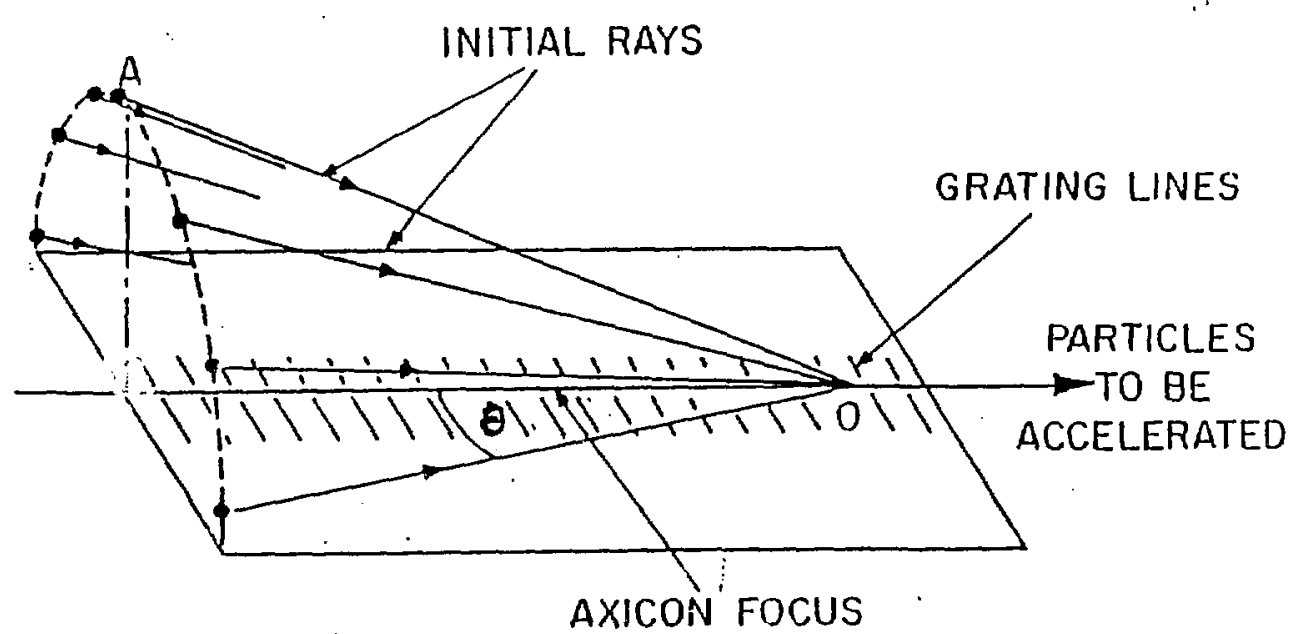

Fig. 3 General geometry for incident waves to have synchronous interaction with a particle. $\theta$ :space angle of radiation with respect to particle direction[5].

Substituting and using the relation $k=2 \pi / \lambda$, where $\lambda$ is the wavelength of the incident radiation, we find that synchronism requires

$$
\lambda=\frac{D}{n}\left(\frac{1}{\beta}-\cos \theta\right)
$$

The synchronism is maintained for incident radiation anywhere (except the $y-z$ plane) in a series of cones around the beam axis. Smith and Purcell discovered in 1952 that charged particles passing over the grooves of a grating cause the emission of electromagnetic radiation[6]. The spectral relation observed for this 
radiation $[7,8]$ is exactly the synchronism condition given in Eq. 25 above, showing that acceleration over true grating surfaces and Smith-Purcell radiation are inverse processes.

For relativistic particle $(\beta=1)$ the synchronism condition can be written

$$
\begin{aligned}
& q_{n}=P \\
& I_{n}=k
\end{aligned}
$$

Now consider the case when $R=0$. This means that the direction of the incident radiation is perpendlcular to the particle beam. In this case the synchronism condition is

$$
\frac{2 \pi n}{D}=k
$$

and we have the relation

$$
D=n \lambda
$$

We are going to restrict our discussions in the remainder of this report to the case where the incident radiation is perpendicular to the particle beam axis $(i$.e. the case $R=0)$. The main reason for this is one of experimental convenience. For this case the optics can form a line focus of the incident radiation along the particle trajectory as shown in Fig. 4. There is in addition a considerable mathematical simplification. The fields over a given grating are then functions of only two variables, the polarization and azimuthal angle $\phi$ of the incident wave. 


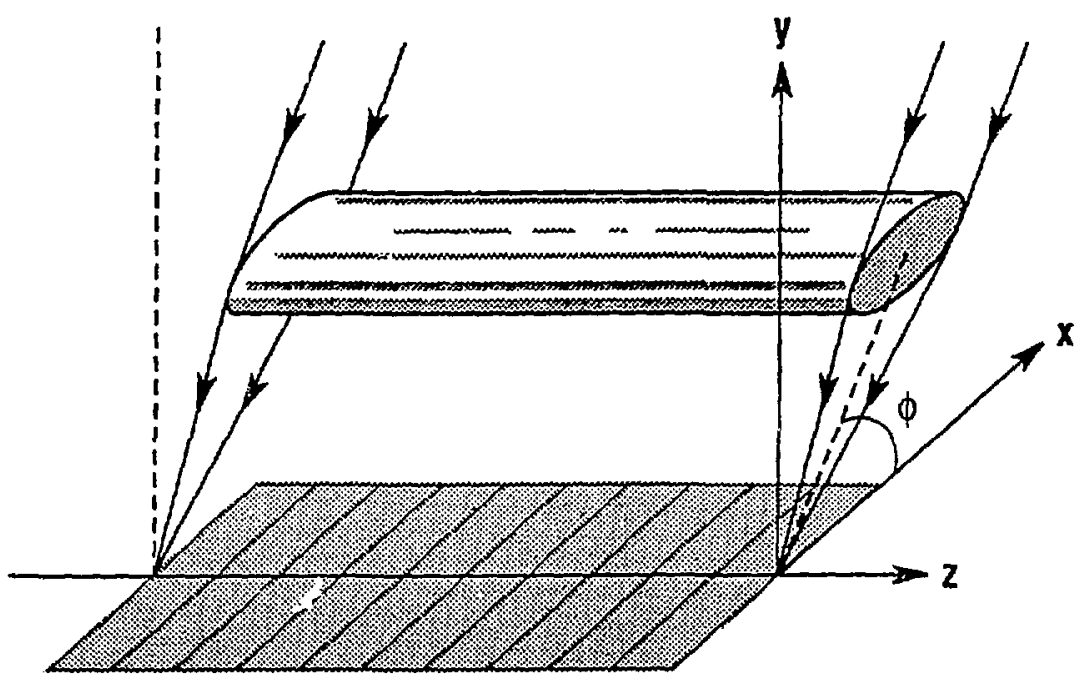

Fig. 4 Cylindrical excitation of the grating accelerating mode using line-focused incident radiation at the angle $\phi$.

\section{Traveling wave field components}

We have seen in section 2 that the field components along the $x$ direction of the space harmonics for the two fundamental states of polarization have the form

$$
F_{x D}^{D}=f_{D} e^{i P x} e^{i q_{D} y} e^{i I_{0} z} e^{-10 t}
$$

Because of the uniformity of the structure along $x$ all the other components must also have an $x$ dependence of $\exp \{i P x\}$. All the components must have an identical $\exp \{-i \omega t\}$ time dependence since we are looking for steady state solutions. Then using the $y$ and $z$ components of the two Maxwell curl equations, it is possible to express all the traveling wave field components in terms of $E_{x}$ and 
$H_{x}$. The results for the two polarizations are:

H-POLARIZATION

$$
\begin{aligned}
& H_{x n}=h_{n} e^{i P x} e^{i q_{n} y} e^{i I_{n} z} e^{-1 \omega t} \\
& E_{x n}=0 \\
& E_{y n}=\frac{-\omega \mu_{0} I_{n}}{Q^{2}} H_{x n} \\
& E_{2 n}=\frac{\omega \mu_{0} g_{n}}{Q^{2}} H_{x n} \\
& H_{y n}=\frac{-P q_{n}}{Q^{2}} H_{x n} \\
& H_{x n}=\frac{-P I_{n}}{Q^{2}} H_{x n}
\end{aligned}
$$

where $Q^{2}=k^{2}-P^{2}$.

E-POLARIZATION

$$
\begin{aligned}
& E_{x n}=\theta_{n} e^{i P x} e^{i \sigma_{n} y} e^{i r_{a} x} e^{-i \omega t} \\
& E_{y n}=\frac{-P q_{a}}{Q^{2}} E_{x m} \\
& E_{x n}=\frac{-P Y_{n}}{Q^{2}} E_{x n} \\
& H_{x m}=0 \\
& H_{y n}=\frac{\omega e_{0} I_{n}}{Q^{2}} E_{x p m} \\
& H_{x D}=\frac{-\omega \epsilon_{a} Q_{n}}{Q^{2}} E_{x D}
\end{aligned}
$$

\section{Shallow sinusoidal grating}

We now want to derive an expression for the strength of the accelerating mode. We consider a grating with surface profile given by

$$
y=h \cos (G z)=h \cos \left(\frac{2 \pi z}{D}\right)
$$

We assume that $h$ is small compared to $D$ so that we have a small ripple on a basically flat surface. We will only consider the case when the incident wave is perpendicular to the particle trajectory (i.e. $R=0$ ). 


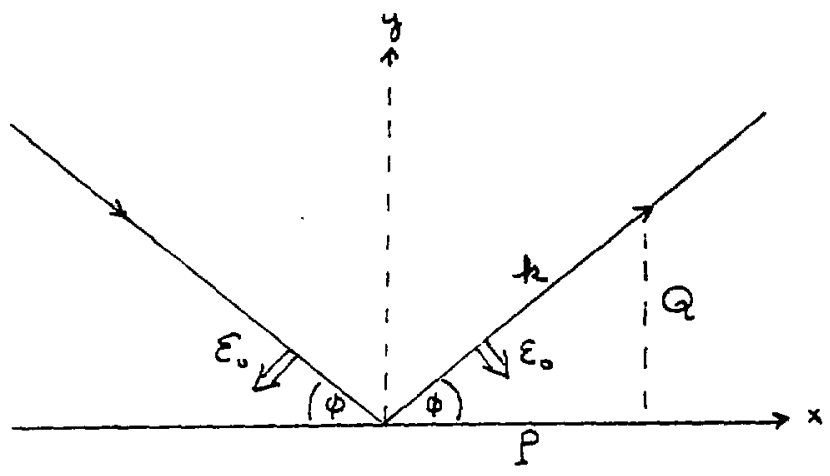

Fig. 5 Incident and reflected wave for E-polarization. The double arrow shows the electric field vector.

E-POLARIZATION

Fig. 5 shows the incident and reflected wave for the case of $E-$ polarization. The boundary condition gives

$$
\begin{gathered}
E_{x}^{I N}+E_{x}^{D}=0 \\
E_{x}^{I N}+E_{x_{1}-1}^{D}+E_{x, 0}^{D}+E_{x_{1} 1}^{D}=0
\end{gathered}
$$

where we neglect the higher space harmonics because of the flatness of the surface. Cancelling the common exp $\{i p x-i \omega t\}$ dependence, the boundary equation becomes

$$
\begin{gathered}
-E_{0} \sin \phi e^{-i \rho y_{0}}+e_{0} e^{i \rho y_{0}}+e_{-1} e^{-P y_{0}} e^{-1 G s} \\
+e_{1} e^{-P y_{0}} e^{1 G x}=0
\end{gathered}
$$

where $y_{S}$ is the value of $y$ evaluated on the grating surface. Since the only outgoing radiated wave is the reflected one, we have

$$
e_{0}=E_{0} \sin \phi
$$

In addition since the structure is perfectly left-right symmetric 
to the incident wave, we must have $e_{-1}=e_{1}$. This leads to the condition

$$
i E_{0} \sin \phi \sin \left(O y_{s}\right)+e_{1} e^{-P y_{0}} \cos (G z)=0
$$

This relation must hold for any value of $z$. If we evaluate it at $z=0$, we find

$$
e_{1}=-i E_{0} O h \sin \phi \Theta^{p h}
$$

The travelling wave equations for E-polarization give

$$
E_{x 1}=\frac{P I_{1}}{Q^{2}} E_{x 1}
$$

Using the conditions for relativistic synchronization, we find that the strength of the accelerating mode is

$$
\frac{E_{s 1}}{E_{0}}=2 \pi \frac{h}{D} \cos \phi
$$

We see that the strength of the accelerating field is directly proportional to the groove depth for shallow gratings. The accelerating field vanishes at $\phi=90^{\circ}$, in accord with Lawson's Theorem. The maximum acceleration is predicted to occur for waves incident close to the grating surface. Fig. 6 shows a rigorous solution[9] to the boundary value problem via an integral equation method using the computer code GERTY. 


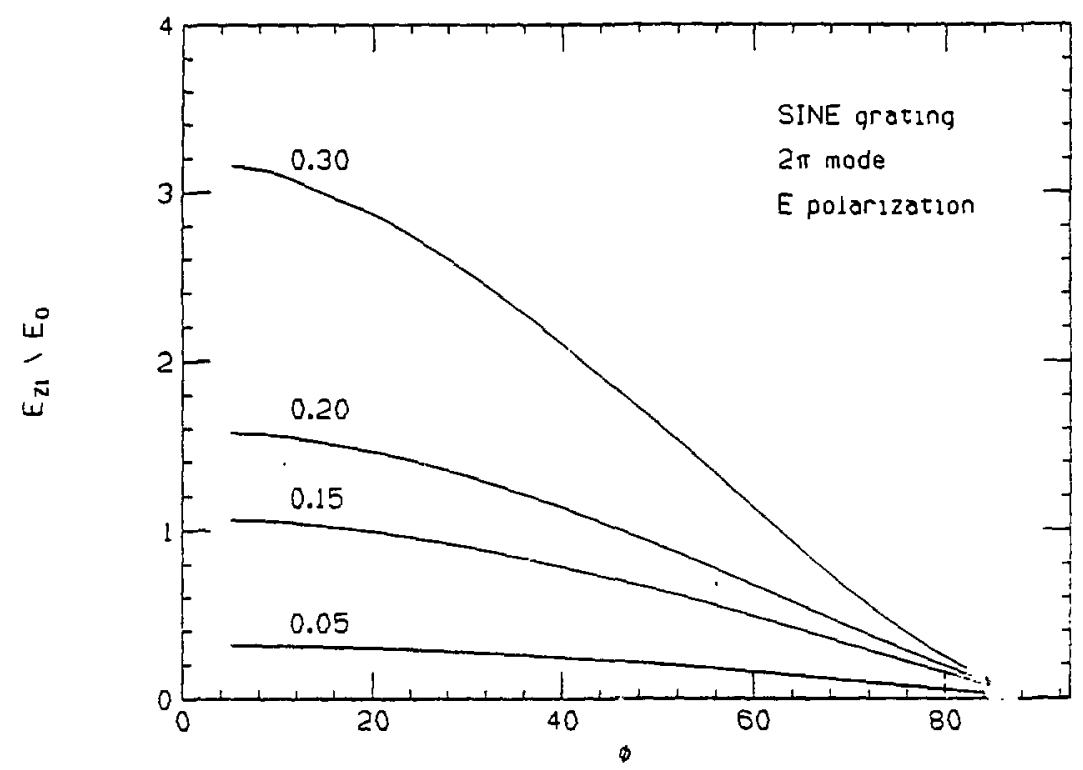

Fig. 6 Accelerating field enhancements versus cylindrical angle $\phi$. The parameters on the curves refer to the ratio $h / D$.

The calculated angular dependence agrees well with Eq. 39. It gives the absolute magnitude of the field correct to within $20 \%$ for $h / D$ up to 0.20 . Note that for $h / D=0.3$ the accelerating field has a strength equal to over three times the magnitude of the incident wave.

H-POLARI ZATION

Fig. 7 illustrates the incoming and reflected waves for the case of H-polarization. 


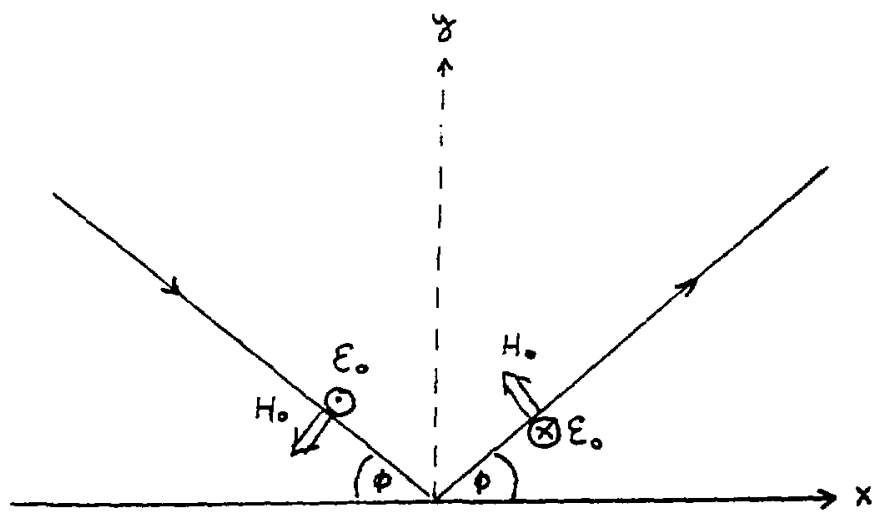

Fig. 7 Incident and reflected waves for H-polarization.

The boundary condition is

$$
\frac{\partial H_{x}^{I N}}{\partial n}+\frac{\partial H_{x}^{D}}{\partial n}=0
$$

We need an expression for the normal derivative at any point on the sinusoidal boundary. Write the position vector from the origin to an arbitrary location on the grating surface as

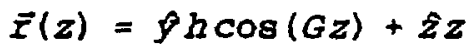

Then the tangent vector at that location is

$$
\frac{d \vec{I}}{d z}=-g h G \sin (G z)+2
$$

Apart from a normalization factor that will cancel out in the 
boundary equation, the normal vector vector at the same location is

$$
\begin{aligned}
\hat{n} & =\frac{d \vec{r}}{d z} \times \hat{x} \\
& =\hat{\rho}+2 h G \sin (G z)
\end{aligned}
$$

The normal derivative can be written in the form

$$
\begin{aligned}
\frac{\partial H}{\partial n} & =\hat{n} \cdot \nabla H \\
& =\hat{n} \cdot\left(\rho \partial_{y}+2 \partial_{z}\right) H
\end{aligned}
$$

If we again restrict the diffracted field to be given by the reflected wave and the \pm 1 evanescent waves, the boundary condition reduces to the relation

$$
H_{0} Q \sin \phi \sin \left(Q y_{0}\right)-h_{1} P e^{-P y_{a}} \cos (G z)-h_{1} G^{2} h e^{-P y_{0}} \sin ^{2}(G z)=0
$$

This is valid for any $z$. However, if we pick certain values like 0 or $\mathrm{D} / 4$, one of the terms in the expression vanishes. To test the general validity of the equation we arbitrarily evaluate at the point $z=D / 8$, so that all the terms will be present. Then for small $y$ we find the coefficient

$$
h_{1}=\frac{H_{0} Q^{2} h \sin \phi}{P+\frac{2 \sqrt{2} \pi^{2} h}{D^{2}}}
$$

The accelerating field is given by Eq. 30

$$
E_{z 1}=\frac{\omega \mu_{0} q_{1}}{Q^{2}} H_{x 1}
$$

for H-polarization. Evaluating this for the case of relativistic synchronization, we obtain finally

$$
\frac{E_{21}}{E_{0}}=\frac{2 \pi \frac{h}{D} \sin \phi \cos \phi}{\cos \phi+\sqrt{2} \pi \frac{h}{D}}
$$

Note that the accelerating field has a more complicated angular dependence than for E-polarization. In addition to vanishing for incident waves coming directly down on the surface, the accelerating field also vanishes for incident waves along the grooves. The factor in the denominator causes the angle corresponding to maximum acceleration to depend on $h / D$. This predicted behavior agrees qualitatively with the results of GERTY calculations[10] shown in Fig. 8. The accelerating field strength is over twice the magnitude of the incident wave for $h / D=0.3$. 


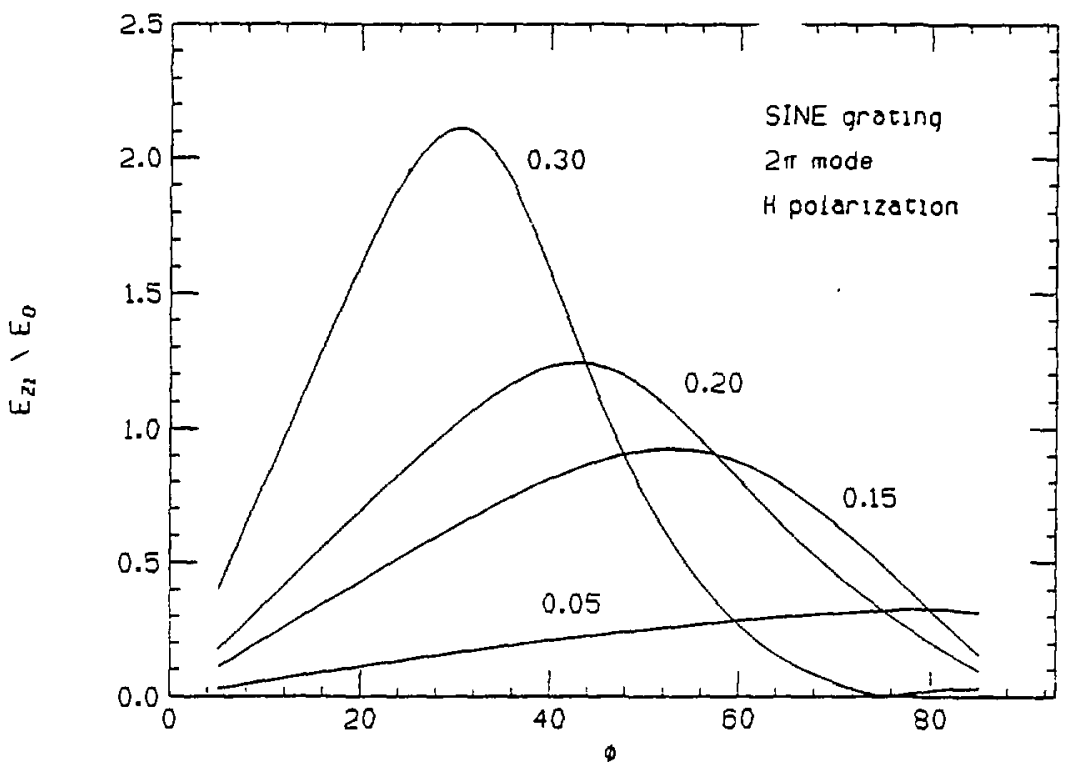

Fig. 8 Accelerating field enhancement versus cylindrical angle $\phi$. The parameters on the curve give $h / D$.

A secondary peak appears for $h / D$ values greater than 0.25 , as we see in Fig. 9 .

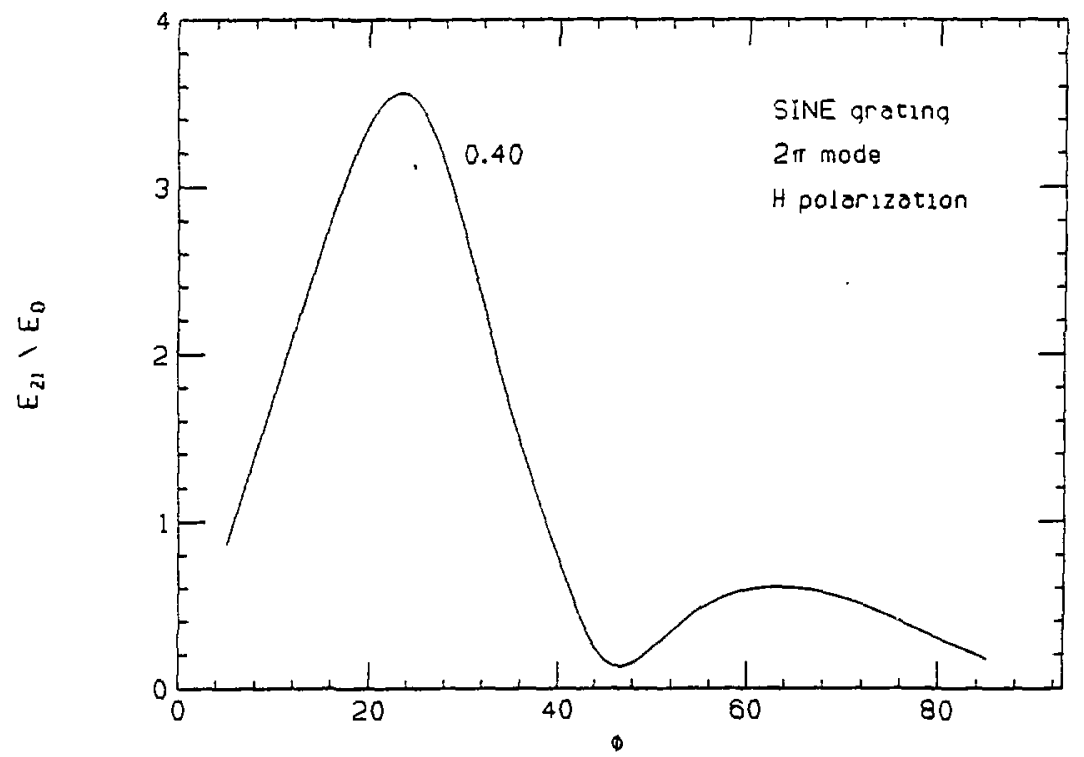

Fig. 9 Enhancement for deep sine grating $(\mathrm{h} / \mathrm{D}=0.4)$ 


\section{6 standing wave fields}

The field components we have discussed so far have all been travelling waves along the $+x$ direction. It will be convenient to consider the case when a second wave travelling in the $-x$ direction is also present. The sum of these two waves will then form standing waves in the $x$ direction. It will then be possible to find nodes along $x$ whare certain field components vanish and where it would be advantageous to position the axis of the accelerated beam. The existence of these nodes allow a practical realization of the grating accelerator where metallic sidewalls Iimit the transverse extent of the grating[11]. Because of the symmetry of the structure we expect the two incident traveliing waves to couple to the structure with the same amplitudes. The form of the field components of the wave travelling along $-x$ is the same as that given previously for $+x$, provided that we substitute $-P$ for $P$ in ail the $\varepsilon$ cuations. Adding the expressions for the two waves we get travelling waves along $z$ with standing wave behavior along $x$.

H-POLARIZATION

$$
\begin{aligned}
& H_{x n}=2 h_{n} \cos (P x) e^{-P y} e^{i\left(x_{n} z-\omega t\right)} \\
& E_{x x}=0 \\
& E_{y n}=\frac{-2 h_{n} \omega \mu_{0} I_{n}}{Q^{2}} \cos (P x) e^{-P y} e^{I\left(x_{n} z-\omega t\right)} \\
& E_{z n}=\frac{2 i h_{n} \omega \mu_{0} P}{Q^{2}} \cos (P x) e^{-P y} e^{i\left(I_{n} z-\omega t\right)} \\
& H_{y n}=\frac{2 h_{n} P^{2}}{Q^{2}} \sin (P x) e^{-P y} e^{i\left(x_{n} z-\omega t\right)} \\
& H_{z n}=\frac{-2 i h_{n} I_{n} P}{Q^{2}} \sin (P x) e^{-P y} e^{i\left(x_{n} z-\omega t\right)}
\end{aligned}
$$

At the $x$ locations where $E_{\text {in }}$ is a maximum the other nonvanishing field components are $H_{\mathrm{rn}}$ and $E_{\mathrm{yn}}$.

E-POLARIZATION

$$
\begin{aligned}
& E_{x n}=2 e_{n} \cos (P x) e^{-P y} e^{1\left(I_{n} z-\omega t\right)} \\
& E_{y n}=\frac{2 e_{n} P^{2}}{Q^{2}} \sin (P x) e^{-P y} e^{i\left(I_{n} z-\omega t\right)} \\
& E_{z n}=\frac{-2 i e_{n} P r_{n}}{Q^{2}} \sin (P x) e^{-P y} e^{i\left(I_{n} z-\omega t\right)} \\
& H_{x n}=0 \\
& H_{y n}=\frac{2 e_{n} \omega e_{0} I_{n}}{Q^{2}} \cos (P x) e^{-P y} e^{i\left(x_{n} z-\omega t\right)} \\
& H_{x D}=\frac{-2 i \theta_{n} \omega e_{0} P}{Q^{2}} \cos (P x) e^{-P y} e^{i\left(I_{n} z-\omega t\right)}
\end{aligned}
$$


At the $x$ locations where $E_{g n}$ is a maximum the only other nonvanishing field component is $E_{\mathrm{gn}}$.

Finally for discussions of the grating surface profile it is convenient to have a total standing wave solution of the fields above the grating surface. Every space harmonic travelling along $+z$ is accompanied by another harmonic of the same order travelling along $-z$. Again because of the symmetry of the structure the two waves should both couple with the same amplitude. We can write the expressions for the wave travelling along $-z$ by substituting $-r_{n}$ for $r_{n}$ in the previous equations. We then add the two travelling waves and use the constraints for relativistic synchronization to obtain the following expressions.

H-POLARIZATION

$$
\begin{aligned}
& H_{x n}=4 h_{n} \cos (P x) e^{-P y} \cos (k z) \cos (\omega t) \\
& E_{x a}=0 \\
& E_{y n}=\frac{-4 h_{n} \omega \mu_{0} k}{Q^{2}} \cos (P x) \theta^{-P y} \sin (k z) \sin (\omega t) \\
& E_{z a}=\frac{4 h_{n} \omega \mu_{0} P}{Q^{2}} \cos (P x) e^{-P y} \cos (k z) \sin (\omega t) \\
& H_{y p}=\frac{4 h_{n} P^{2}}{Q^{2}} \sin (P x) e^{-P y} \cos (k z) \cos (\omega t) \\
& H_{z n}=\frac{4 h_{n} P k}{Q^{2}} \sin (P x) e^{-P y} \sin (k z) \cos (\omega t)
\end{aligned}
$$

Fig. 10 shows the the electric field pattern for the $n=1$ accelerating mode at several instants of time. Note that the diffracted field must contain in addition other space harmonics so that the boundary conditions can be satisfied by the total field. In particular the addition of the incident field to the accelerating mode tends to reinforce the field inside the grooves and cance 1 the field above the ridges. For H-polarization the field lines jump from one ridge to the next over the grooves of the grating. Note that all the field lines are pointing in the same direction. It is easy to see that this configuaration can couple to an incident plane wave in the $x-y$ plane. Also shown are the positions of a particle with the proper phase to receive continuous acceleration. We will refer to this case as the $2 \pi$ accelerating mode. 


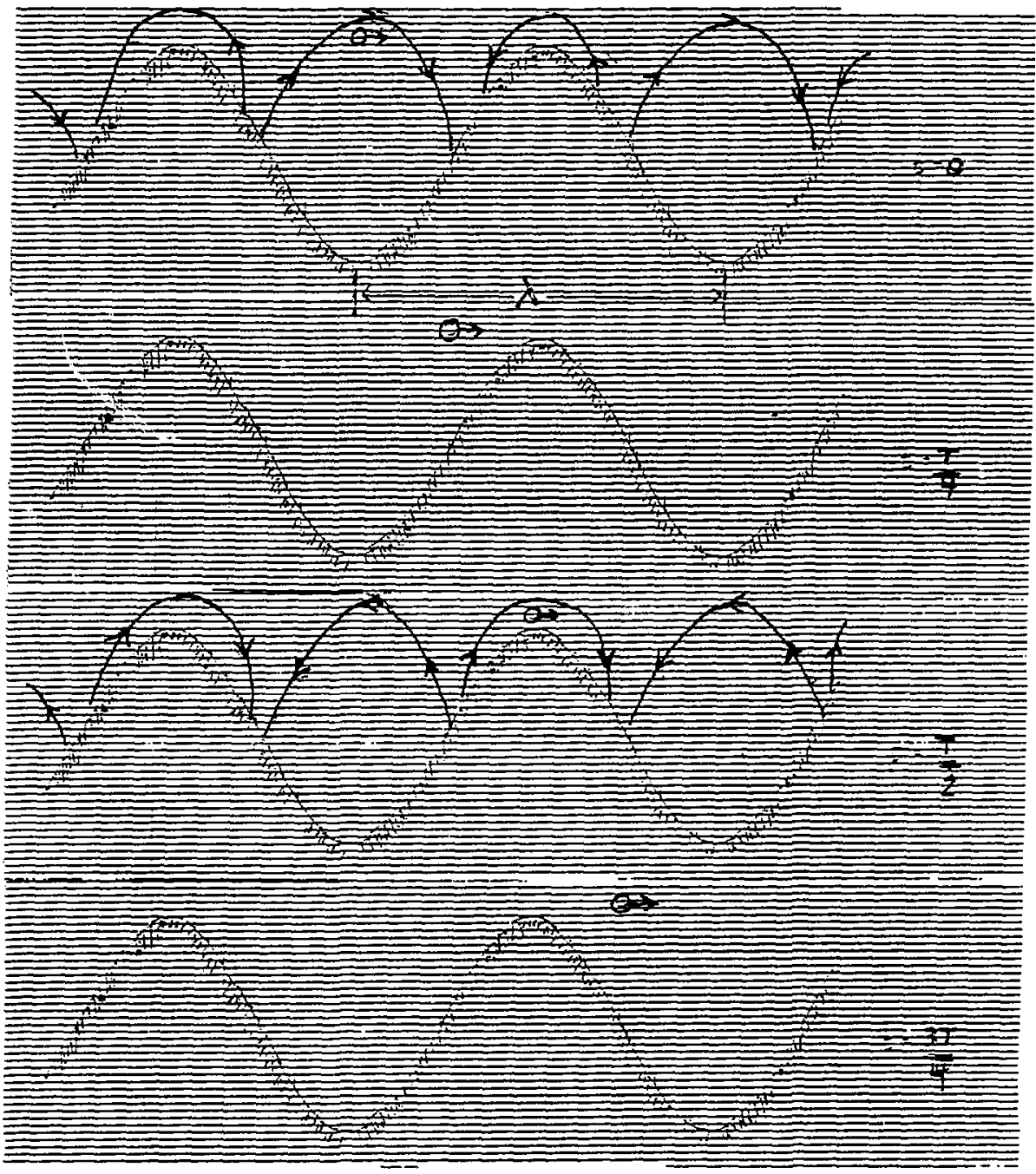

Fig. 10 Electric field pattern due to the accelerating mode for H-polarization. The field lines and a test particle are shown for the time equal to quarter periods. 
E-POLARIZATION

$$
\begin{aligned}
& E_{x=0}=4 e_{n} \cos (P x) e^{-P y} \cos (k z) \cos (\omega t) \\
& E_{y n}=\frac{4 e_{n} P^{2}}{Q^{2}} \sin (P x) e^{-P y} \cos (k z) \cos (\omega t) \\
& E_{z n}=\frac{4 e_{n} k P}{Q^{2}} \sin (P x) e^{-P y} \sin (k z) \cos (\omega t) \\
& H_{x n}=0 \\
& H_{y n}=\frac{4 e_{n} \omega e_{0} k}{Q^{2}} \cos (P x) e^{-P y} \sin (k z) \sin (\omega t) \\
& H_{z n}=\frac{-4 e_{n} \omega e_{0} P}{Q^{2}} \cos (P x) e^{-P y} \cos (k z) \sin (\omega t)
\end{aligned}
$$

The $2 \pi$ accelerating mode patterns for E-polarization are shown in Fig. 11. In this case the electric field lines originate on the floor of the grooves. Again note that the direction of the field lines are the same in each period so that it can couple to an incident plane wave and that a particle with the proper phase can be continously accelerated.

These standing wave solutions point out one of the major problems with using a true grating as an accelerator cavity. The accelerating field exists over the entire surface of the grating. This leads to a large inefficiency since one is only using the field in a narrow band near the particle axis. To solve the problem one is forced to add side walls at nodes of the field pattern along $x$. This requirement compromises two of the main advantages of the true grating, namely its simplicity of construction and its openness to the external radiation field.

\section{Forces}

We would now like to consider the net force seen by a particle with a given phase relative to the incident radiation. We define the net value of a force component to be

$$
\left\langle F_{i}\right\rangle=\frac{1}{T} \int_{-T / 2}^{T / 2} F_{i}(t) d t
$$

where $T=D / c$ is the time required for a relativistic particle travelling along $z$ to cross one period of the structure. Let $z_{0}$ be the $z$ location of a particle at $t=0$. Then we can define the reference particle to be the one with $z_{0}=0$. 


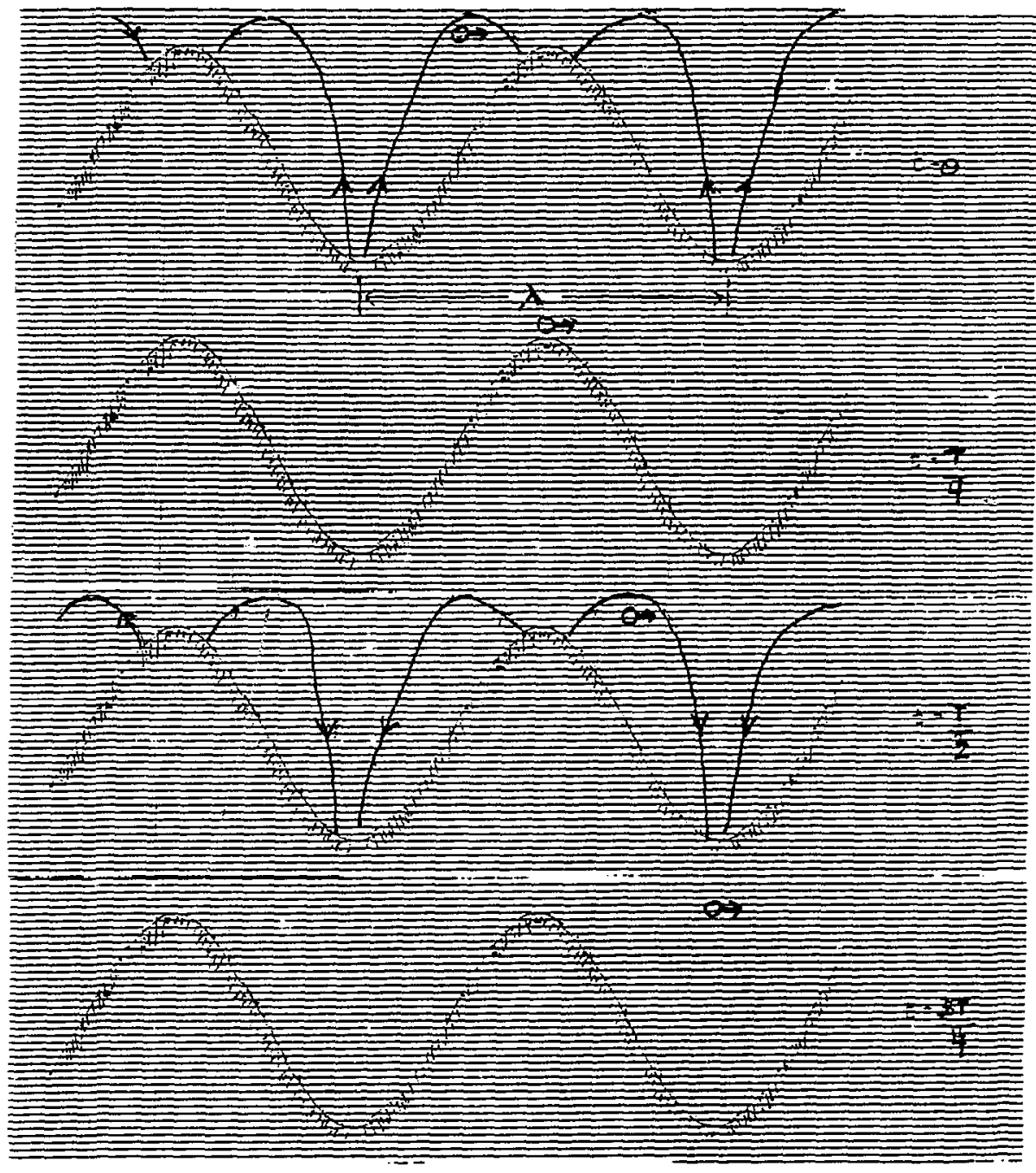

Fig. 11 Electric field pattern due to the accelerating mode for E-polarization. The field lines and a test particle are shown for the time equal to quarter periods. 
The other particles have a phase with respect to the reference particle given by

$$
\Phi=2 \pi \frac{z_{0}}{D}
$$

The $z$ dependence of an arbitrary particle can then be written

$$
z(t)=c t+\frac{D}{2 \pi} \Phi
$$

The force components acting on a particle at time $t$ are

$$
\begin{aligned}
& F_{x n}(t)=q\left[E_{x n}(t)-c \mu_{0} H_{y n}(t)\right] \\
& F_{y n}(t)=q\left[E_{y n}(t)+c \mu_{o} H_{x n}(t)\right] \\
& F_{z n}(t)=q E_{z n}(t)
\end{aligned}
$$

The time averages of these expressions remove the explicit $z$ and $t$ dependence and introduce the factor $\Phi$. For example

$$
\langle\cos [k z(t)] \cos [\omega t]\rangle=\frac{1}{2} \cos (\phi)
$$

The results of the calculations for H-polarization are

$$
\begin{aligned}
& \left\langle F_{x x}\right\rangle=q E_{s} \frac{P}{k} \sin (P x) e^{-P y} \cos (\Phi) \\
& \left\langle F_{y x}\right\rangle=q E_{g} \frac{P}{k} \cos (P x) e^{-P y} \cos (\phi) \\
& \left\langle F_{x p}\right\rangle=q E_{S} \cos (P x) e^{-P y} \sin (\Phi)
\end{aligned}
$$

where the effective accelerating field $E_{S}$ at the surface is given by

$$
E_{S}=-2 h_{L} C \mu_{\circ} \frac{P k}{Q^{2}}
$$

It is possible to write the net force components for E-polarization in the same form given above, provided that the beam axis is shifted by a quarter wavelength along $x$, and that we define the effective accelerating field as

$$
E_{s}=2 e_{\mathrm{a}} \frac{P k}{Q^{2}}
$$

Examining these equations we see that all force components decrease exponentially with distance away from the grating surface. Particles traveling on the beam axis see no net force in the $x$ direction. However, particles on the beam axis do see a net vertical deflecting force. Fortunately, the deflecting forces are out of phase with the accelerating force, so the deflecting force 
would be small for a short bunch centered around the accelerating phase. In addition the strength of the deflecting forces is reduced by a factor $\mathrm{P} / \mathrm{k} \approx 0.3$ relative to the accelerating force.

The nonvanishing vertical force on any bunch with a finite length is one of the major difficulties with using true gratings as accelerating structures. Firstly, it complicates the construction of the accelerator because some method must be provided to get rid of it. Some of the suggested methods are:

(1) We can provide an external magnetic field along the entire length of the accelerator. The field is directed along the grooves to give an additional deflecting force exactly canceling the force from the grating itself[5]. However, a given external field strength can only compensate the deflection on a particle at a given value of $x, y$, and $\Phi$. There will always be a net residual deflection on some particles in a finite sized bunch.

(2) We can rotate sections of grating by $90^{\circ}$ every so often down the length of the accelerator[11]. Then a particle subject to a positive vertical force, for example, would see a positive horizontal force, then a negative $y$ force, then a negative $x$ force, etc. This scheme increases the structural complexity and alignment difficulties for the accelerator. In addition, it restricts the "openness" of the device.

(3) We can purposely add discontinuities in the groove spacing every so often down the length of the accelerator. This corresponds to periodically changing a particle's phase. It is known that this method can be used to provide strong focusing in a grating accelerator $[12]$. Depending on the requirements, it may also suffice for controlling the particles' deflection. This scheme could be done, for example, by mounting each section of accelerator on a precision translation stage.

The second problem with the non-vanishing y component of the force is that particles in the bunch are always subject to synchrotron radiation losses. Since these losses grow like $\gamma^{2}$, they eventualiy set an upper limit on the energy that can be reached using this type of structure.

\section{$8 \mathrm{Pi}$ mode accelerating structures}

The structures we have discussed up to now have had a phase advance of $2 \pi$ per period. Palmer[5] has proposed using a mode structure, as shown in Fig. 12. The advantage of a $\pi$ mode structure is that it is possible in principle to obtain an infinite accelerating field enhancement for the case of an infinitely conducting grating. In a unique approach Palmer begins by specifying the combination of space harmonics that would produce a useful accelerator structure. 

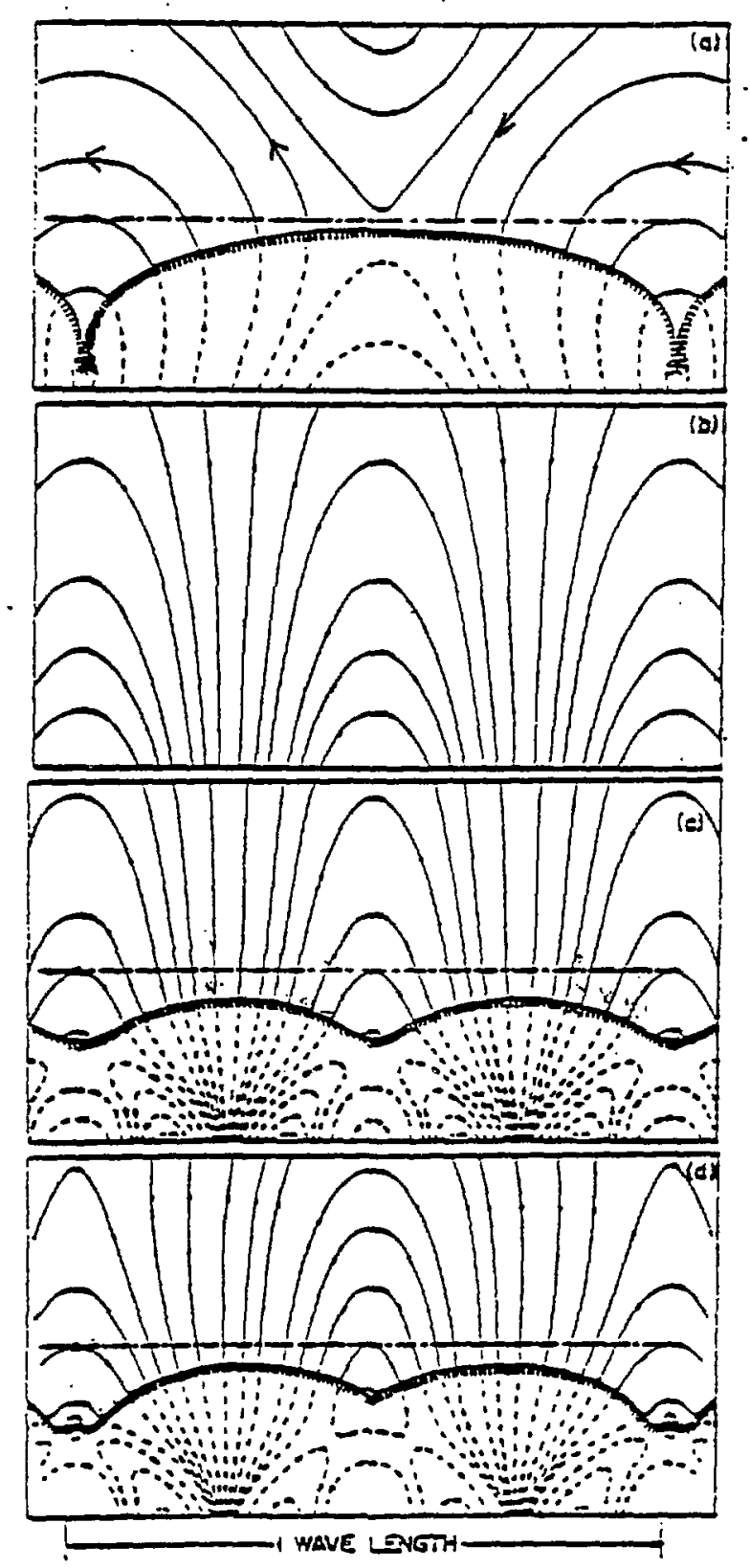

Fig. 12 Standing wave electric field lines due to various space harmonics[5]. (a) $n=0$ plus $n= \pm 1$ modes; (b) $n= \pm 1$ modes only; (c) pure $\pi$ mode; (d) $\pi$ mode. 
He then finds the corresponding surface boundary by requiring that it be perpendicular to the standing wave electric field lines. The boundary required to support a pure accelerating mode $(n=1)$ would require infinitely deep cusps into the surface. Therefore, to obtain a realizable shape he adds a small admixture of the $n=3$ space harmonic to the total field. This produces an acceptable $\pi$ mode accelerating structure, but the structure does not couple to radiation waves in the $x-y$ plane. The easiest way to see this is to imagine that the field over the grating arises from two oppositely directed dipoles oriented along the $y$ direction. For a pure $\pi$ mode the fields from the two dipoles exactly cancel in the $x-y$ plane and hence there is no coupling. This problem can be solved by adding a small component of the $n=0$ space harmonic to the total field. Note that after this is done the period of the structure changes from $D / 2$ to $D$ and thus it is no longer a pure $\pi$ mode. We will refer to this case of a $\pi$ mode structure with a small $2 \pi$ perturbation as a ir mode structure.

We have used GERTY to calculate the accelerating field enhancement for structures with the general surface profile

$$
y(z)=h_{1} \sin \left(\frac{2 \pi z}{D}\right)+h_{2} \sin \left(\frac{4 \pi z}{D}\right)
$$

Fig. 13 shows the enhancement for E-polarization as a function of the azimuthal angle $\phi$ for several values of $\left(h_{1}, h_{q}\right)$.

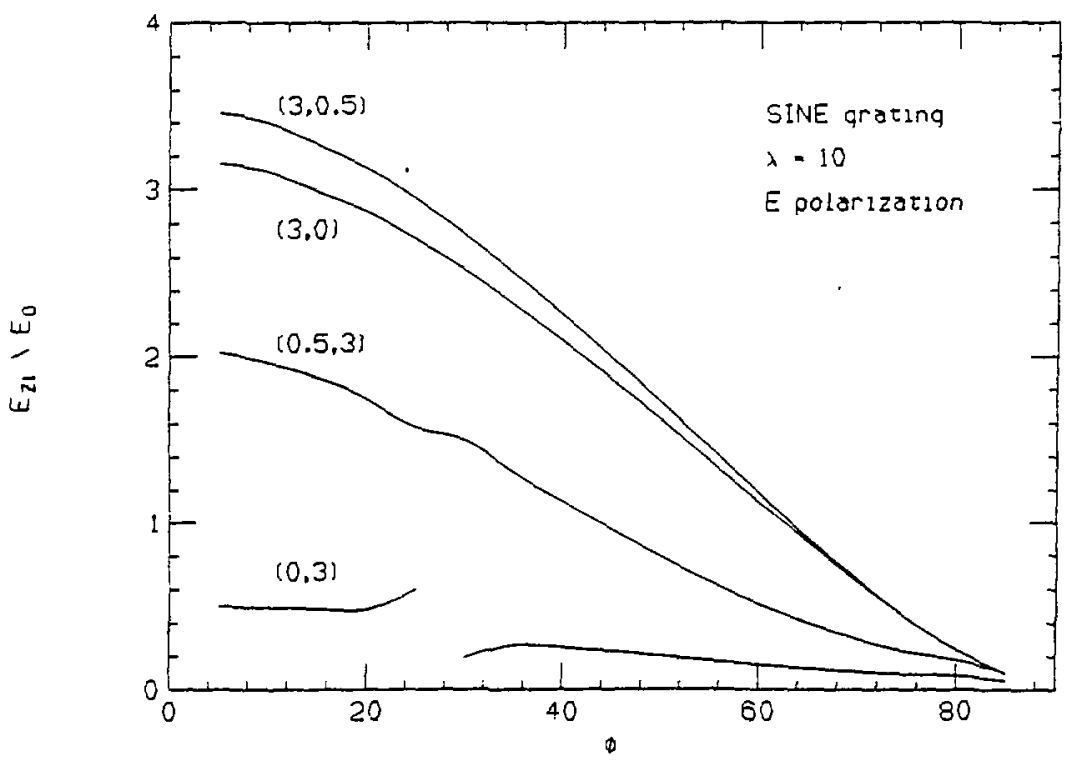

Fig. 13 Accelerating field enhancement versus cylindrical angle $\phi$ for E-polarization. The parameters on each curve refers to the values of $h_{1}$ and $h_{2}$ in Eq. 61 . 
The curve marked $(3,0)$ is an example of the $2 \pi$ mode. The curve marked $(3,0.5)$ shows that adding a small $h_{2}$ term only makes a small change to the enhancement. The pure $\pi$ mode case is marked $(0,3)$. The enhancement indicated here basically shows the level of accuracy of the program for this polarization. The curve for the case $(0.5,3)$ shows that there isn't any enhancement advantage in using a $\pi$ mode in E-polarization.

Similar data for H-polarization is shown in Fig. 14. The curve marked $(3,0)$ shows the pure $2 \pi$ mode. The curve marked $(3,0.5)$ shows that adding a small $h_{2}$ term leads to the addition of a second, narrow peak around $73^{\circ}$. The pure $\pi$ mode case is marked $(0,3)$. The calculated enhancements are very small, indicating the calculation is accurate for H-polarization. The case marked $(0.5,3)$ shows one very strong and narrow $\pi$ enhancement around $27^{\circ}$, as well, as a second much smaller peak near $81^{\circ}$. In general we see that a $\pi^{*}$ mode structure offers the advantage of much larger enhancements over a $2 \pi$ mode structure. The disadvantages are a more complicated surface profile and a much tighter alignment tolerance on the direction of the incoming radiation.

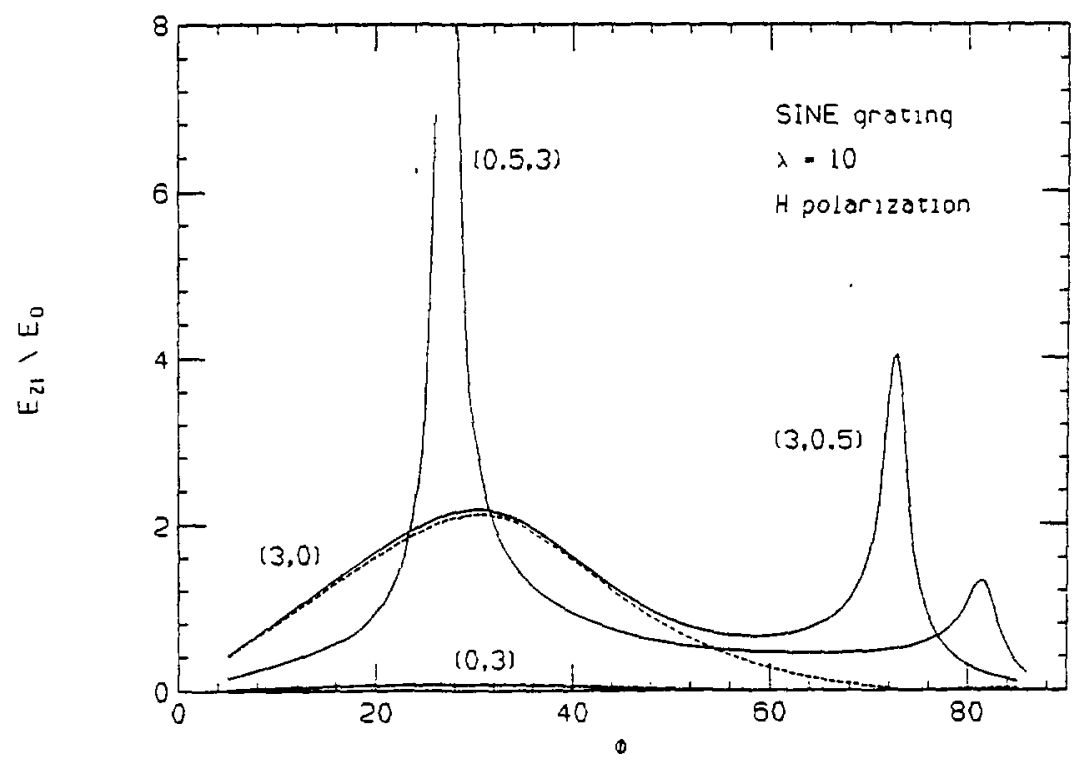

Fig. 14 Accelerating field enhancement versus cylindrical angle $\phi$ for $H$ - polarization. Parameters on each curve refers to the values of $h_{1}$ and $h_{2}$ in Eq. 61 . 


\section{Vee grooved structures}

Up until now we have only considered gratings with a sinusoidal surface profile. However, we will find it advantageous to be able to specify and accurately manufacture gratings with known profiles. One way to do this is to make use of the mature etching technology developed for the manufacture of integrated circuits. It turns out that the depth of an etch along the $\langle 111\rangle$ plane of silicon can be accurately determined. This leads us to examine vee grooved structures[13] with the general shape indicated in Fig. 15. The fixed angle of $54.7^{\circ}$ corresponds to the angle between the $\langle 111\rangle$ and $\langle 100\rangle$ planes in silicon.

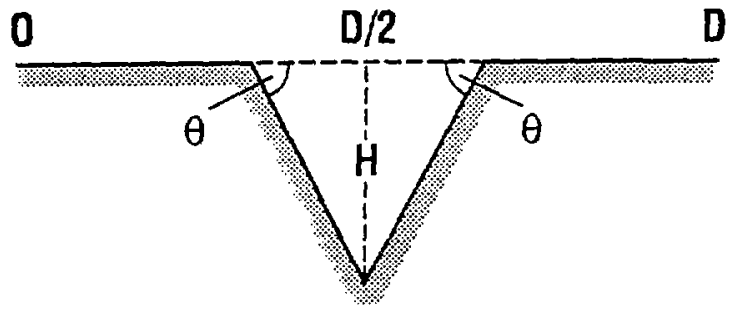

Fig. 15 General shape for $2 \pi$ vee-grooved structure.

$D:$ period: H:depth, $\theta=54.7^{\circ}$.

The $2 \pi$ mode structures have a single vee groove per period. In this case the groove depth $\mathrm{H}$ is the only adjustable parameter. Fig. 16 shows the accelerating field enhancement versus the azimuthal angle $\phi$ for E-polarization. The behavior is similar to that seen in $2 \pi$ sinusoidal gratings, although the enhancement is considerably smaller. The H-polarization results in Fig. 17 is also similar to the sinusoidal case. The angles corresponding to the peak enhancement move towards smeller $\phi$ as the depth of the groove is increased. The magnitude of the enhancement is somewhat smaller than for the sine grating. 


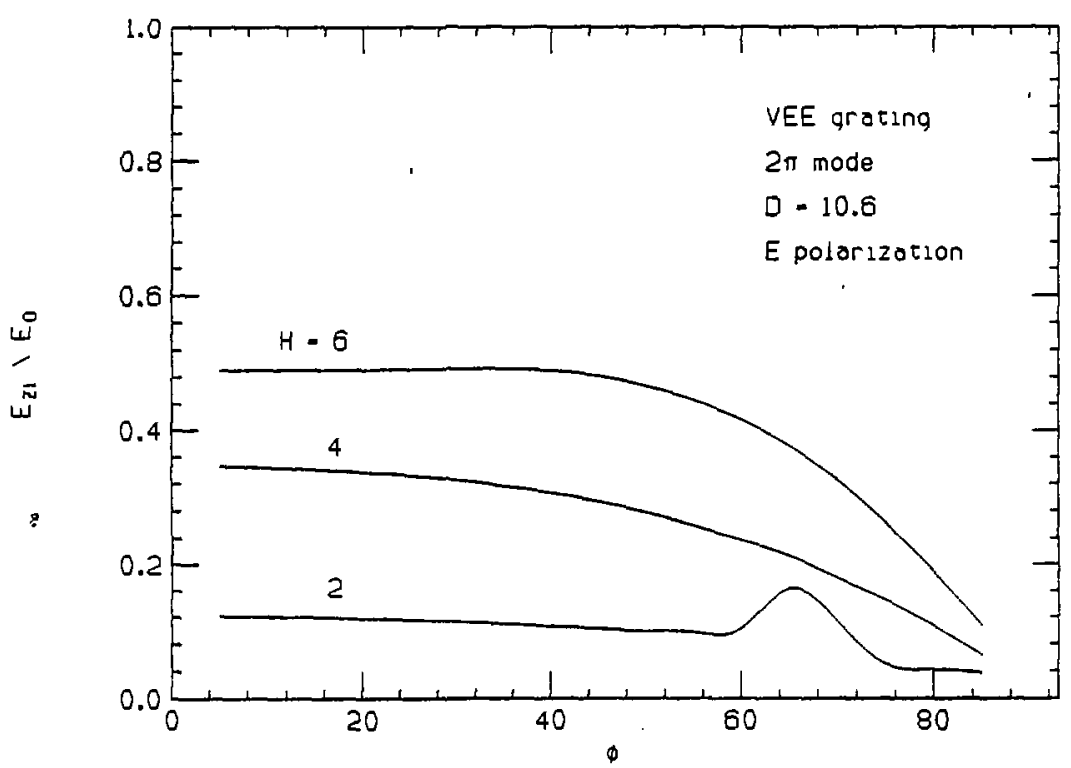

Fig. 16 Accelerating field enhancements versus cylindrical angle $\phi$ for E-polarization.

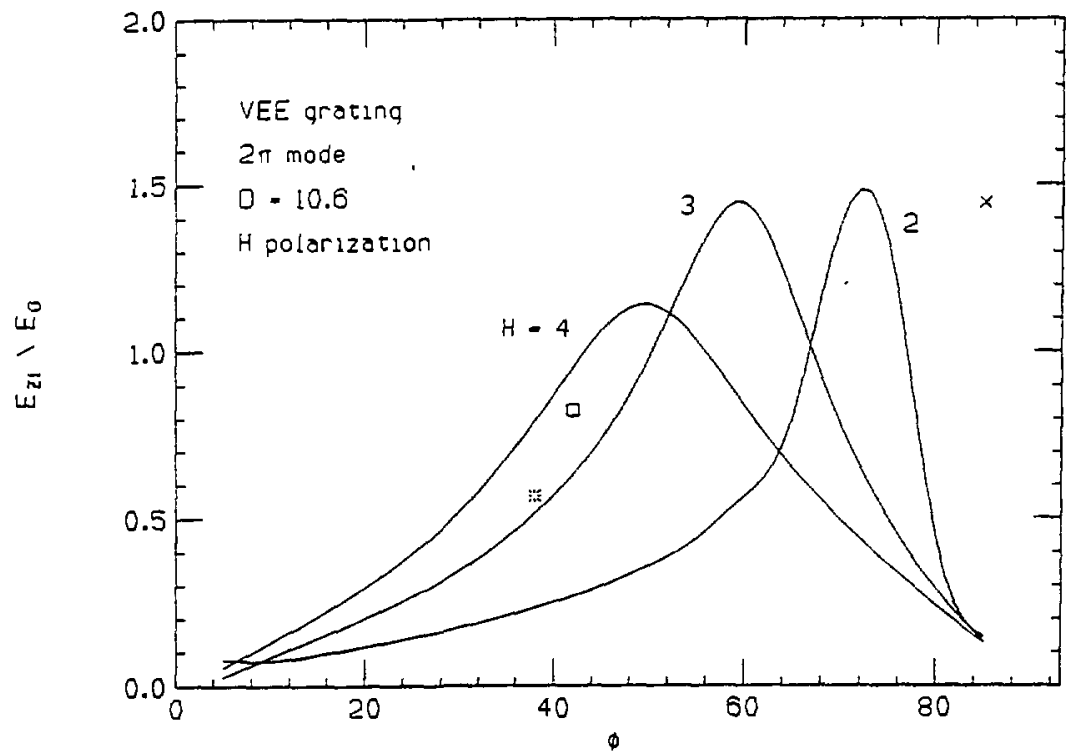

Fig. 17 Accelerating field enhancements versus cylindrical angle $\phi$ for $H$-polarization. $(x)$ peak for $H=1$; (D) peak for $H=5 ;(*)$ peak for $H=6$. 


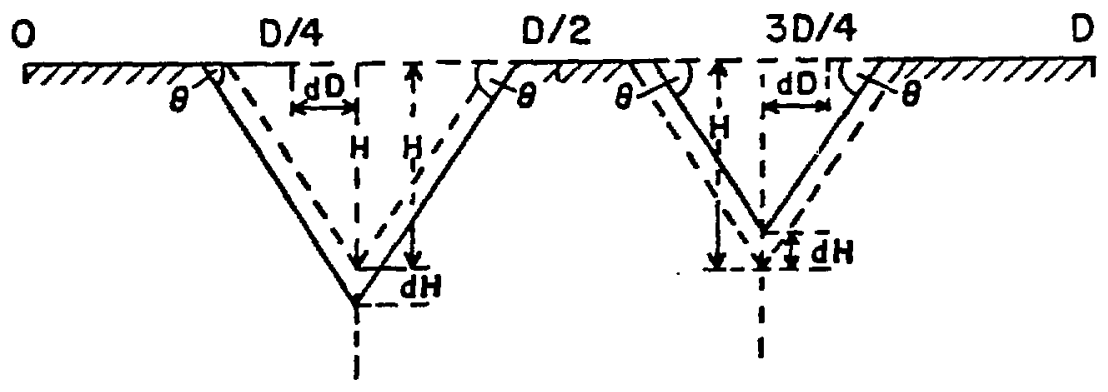

Fig. 18 General shape for vee-grooved structure. D:period, H:mean groove depth, dH:differential groove depth, $\theta=54.7^{\circ}$.

A grating profile that supports $\pi$ and $\pi^{*}$ modes is shown in Fig. 18 . The two parameters here are the mean vee depth $H$ and the differential depth dH between the two grooves.

Results for E-polarization are shown in Fig. 19. Again we see that there is no significant enhancement for this polarization. However, the grating does perform the function of transforming the direction of the electric field of the incoming plane wave, so that it can be used for acceleration. We believe that the small peak near $50^{\circ}$ is due to numerical instabilities specific to E-polarization.

Fig. 20 shows enhancement data for H-polarization and for a fixed value $\mathrm{dH}=0.5$. An enhancement exceeding 4 is observed for a depth $H=3$. Figs. 21 and 22 show that the peaks are larger and narrower for a fixed $H$ when $d H$ is decreased. This is particularly true for the shallower $H=2$ case. Note that Fig. 22 shows that, as expected, there is no enhancement for the pure $\pi$ mode case with $d H$ $=0$. Fortunately, the numerical technique appears more accurate for H-polarization. 
GRATING STRUCTURE

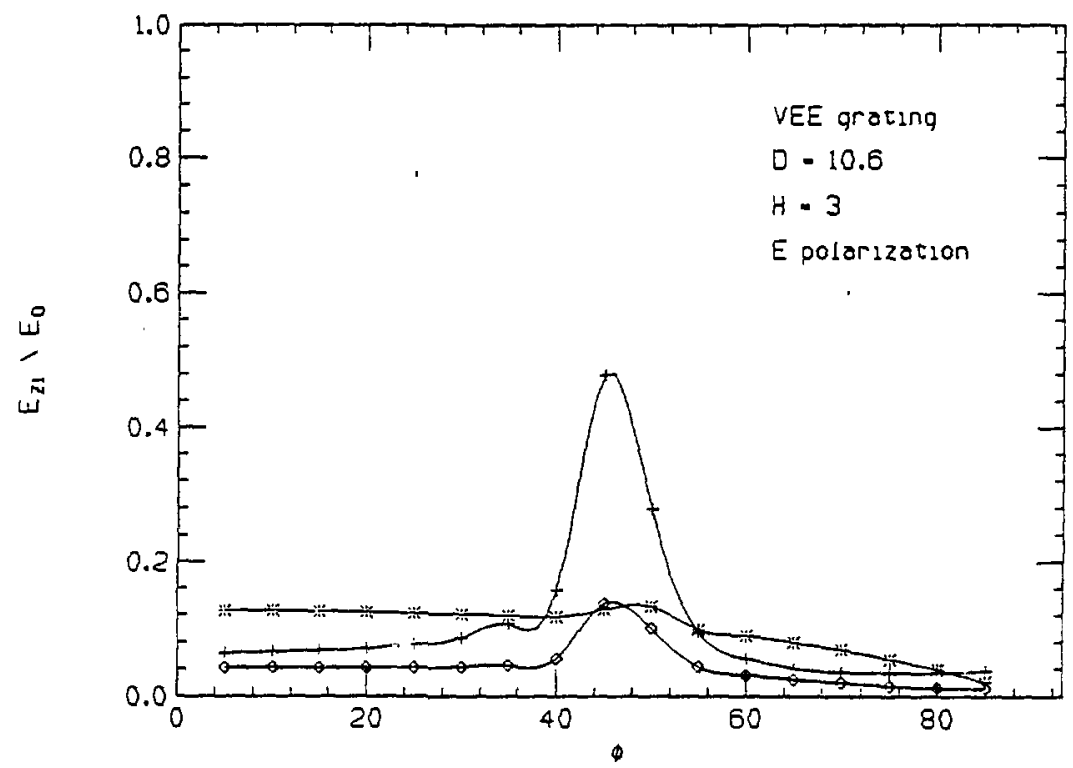

Fig. 19 Accelerating field enhancements versus cylindrical angle $\phi$ for E-polarization. $(+) \mathrm{dH}=0.0$; (*) $\mathrm{dH}=0.5 ;(0) \mathrm{dH}=0.2$.

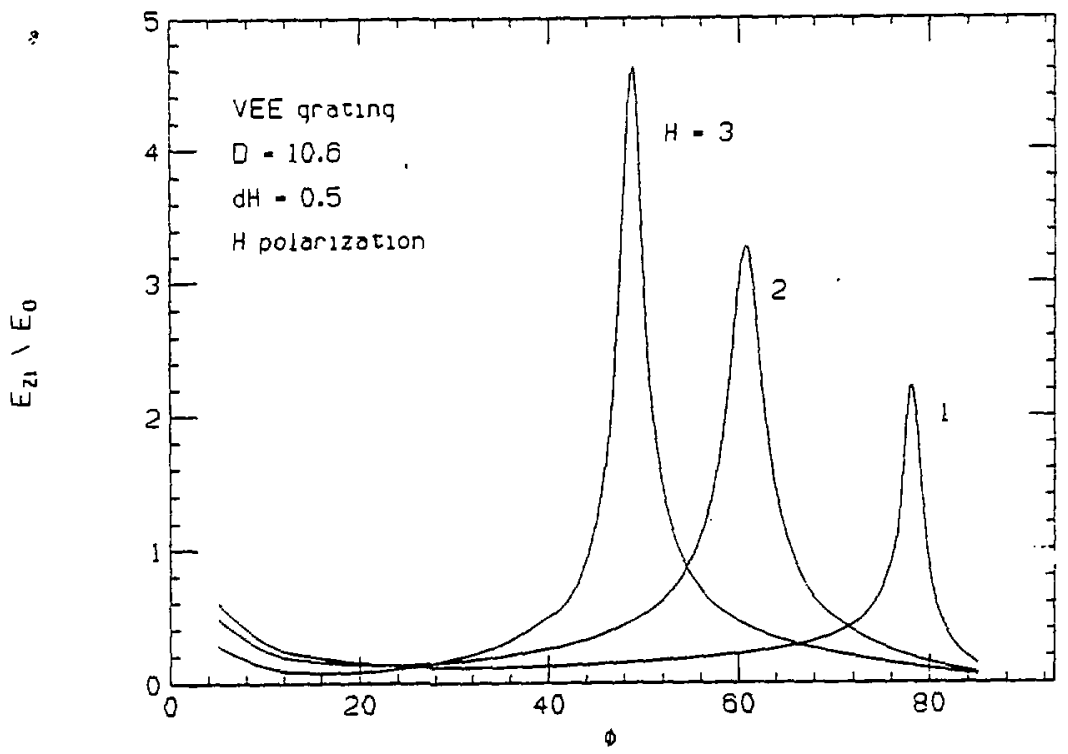

Fig. 20 Accelerating field enhancements versus cylindrical angle $\phi$ for $H$-polarization. 


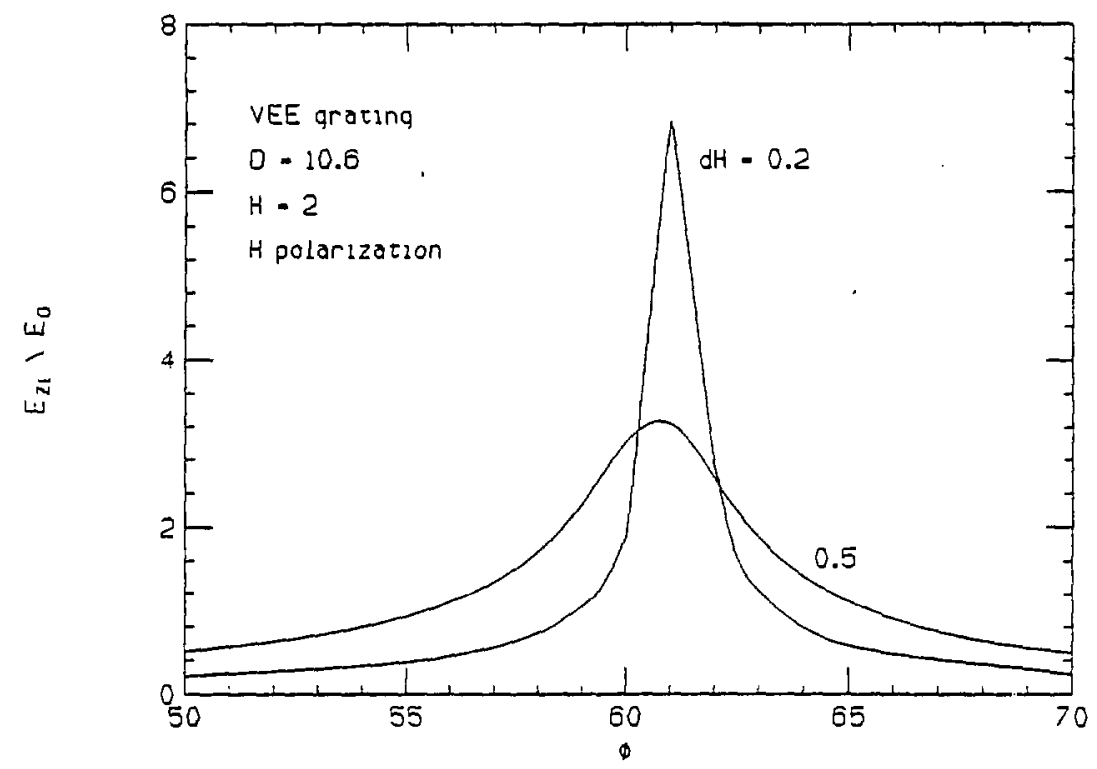

Fig. 21 Accelerating field enhancements versus cylindrical angle $\phi$.

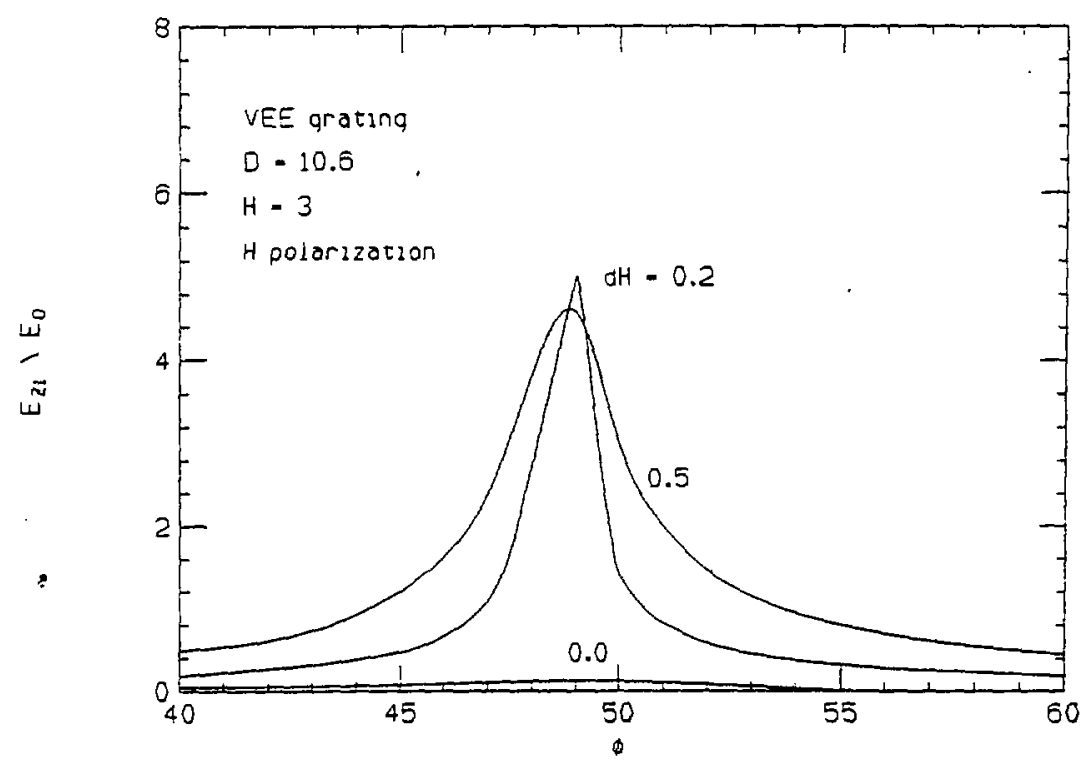

Fig. 22 Accelerating field enhancements versus
cylindrical angle $\phi$. 


\section{Laser linac}

The properties of the grating structures that we have discussed are not restricted to optical wavelengths. The period and other dimensions of any of these structures can be scaled to any wavelength we desire. However, the original impetus for studying these devices was to make use of the large peak power available from lasers. Therefore, let us examine the predicted acceleration using the parameters of an existing laser facility (the Accelerator Test Facility at Brookhaven National Laboratory).

The peak electric field $E_{p}$ is related by the Poynting vector to the time-averaged power density $S$ by

$$
E_{p}=\sqrt{C \mu_{0} S}
$$

Numerically, we have

$$
E_{p}[V / m]=27.5 \sqrt{S\left[W / m^{2}\right]}
$$

Let us consider a carbon dioxide laser $(\lambda=10.6 \mu \mathrm{m})$ with a pulse length of $6 \mathrm{ps}$. Assume we use cylindrical optics with an f-number $f \#=5$ in the focussing direction. The radius of the focussed spot is $\mathrm{r} \approx \mathrm{f} \#=23 \mathrm{\mu m}$. Then we find that we can maintain a peak incident electric field of $1 \mathrm{GV} / \mathrm{m}$ if the laser can supply a pulse energy of $0.84 \mathrm{~J}$ per meter of accelerator length.

The effective accelerating field is then given by

$$
E_{A}=E_{S} K e^{-P y_{0}}
$$

where $x$ is the field enhancement factor and $y_{0}$ is the mean beam height above the grating surface. The factor $x$ depends on the specific surface profile and can, as we have seen, easily be a factor of 4 or more. In a real device the accelerating field will probably be limited by surface damage effects, so the enhancement factor would actually be used to reduce the pulse energy requirement on the laser. To be useful the beam emittance must be small enough to make $\exp \left\{-\mathrm{Py}_{0}\right\} \approx \mathrm{e}^{-l}$.

\section{Grating lens}

Although our emphasis has been on using the grating as an accelerating structure, a more practical application may be as a focussing element. Consider a bunch of particles traveling with velocity $\approx c$ in the $z$ direction. Assume that the particles have previously gone through a bunching stage and that they enter the lens with a phase that maximizes the deflection. Then the 
acceleration per period will be small and we can treat $\gamma$ as approximately constant.

The transverse equations of motion can be then written

$$
\begin{aligned}
F_{x} & =\frac{d}{d t}\left(y m v_{x}\right)=c \frac{d}{d z}\left(m c y \frac{d}{d z} x\right) \\
& =m c^{2} \gamma \frac{d^{2} x}{d z^{2}}
\end{aligned}
$$

with a similar equation for $y$. For particles near the beam axis we can treat the $x$ and $y$ coordinates of the particles as small quantities and the equations of motion become

$$
\begin{aligned}
& m c^{2} \gamma \frac{d^{2} x}{d z^{2}}=q E_{\lambda} \frac{p^{2}}{k} \times \cos (\Phi) \\
& m c^{2} \gamma \frac{d^{2} y}{d z^{2}}=q E_{A} \frac{P}{k}(1-P y) \cos (\Phi)
\end{aligned}
$$

Note that the $x$ equation has the form of a harmonic oscillator. Apart from a constant term, the $y$ equation is also in the form of a harmonic oscillator. For a given phase $\Phi$ we have focussing in one direction and defocussing in the other. The constant vertical force is given by

$$
F_{\text {ext }}=g E_{A} \frac{P}{k} \cos (\Phi)=q C B_{\text {ext }}
$$

In order to keep the particles centered on the lens axis we must. cancel this force. Therefore a constant external magnetic field must be applied along the $x$ direction with the magnitude

$$
B_{\text {ext }}=\frac{E_{\lambda}}{C} \frac{P}{K} \cos (\Phi)
$$

Note that the strength of this magnetic field depends on $\Phi$. It is therefore only possible to exactly cancel the deflection for one value of the phase. Focusing of particles in a finite length bunch will be subject to higher order optical aberrations. After the constant deflection has been removed, both equations have the form 


$$
\frac{d^{2} x}{d z^{2}}=\frac{p^{2} \cos (\phi)}{L_{o} k} x
$$

where the characteristic length $\mathrm{L}_{0}$ is given by

$$
L_{0}=\frac{m c^{2} \gamma}{q E_{A}}
$$

Since $x^{\prime}=\mathrm{dx} / \mathrm{d} z$, we can express this in terms of a thin lens by

$$
\Delta x^{\prime}=\frac{P^{2} \cos (\Phi)}{L_{0} k} \Delta z x
$$

The focal length of the lens is

$$
f=L_{\circ}\left(\frac{k}{P}\right)^{2} \frac{1}{2 \pi \cos (\Phi)} \frac{\lambda}{L_{0}}
$$

where $\lambda$ is the wavelength of the incident radiation and we have used $\Delta z=L_{0}$, the length of the quadrupole. The focal length can be related to the gradient $G$ of the quadrupole through

$$
f=\frac{p}{q G L_{0}}
$$

where $p$ is the momentum of the particle. The gradient is given by

$$
G=2 \pi \frac{B_{A}}{\lambda}\left(\frac{P}{k}\right)^{2} \cos (\Phi)
$$

where the effective "pole tip" field $B_{A}=E_{A} / c$. Note that choosing a large incident angle $\phi$ minimizes the required external magnetic field, but it also reduces the available gradient. The grating profile should be designed to optimize these competing requirements.

Table 1 shows examples of a grating lens powered by a $\mathrm{CO}_{2}$ laser. We assume the radiation is incident normal to the electron beam trajectory. 


\begin{tabular}{|l|l|l|l|}
\hline \multicolumn{2}{|c|}{ Table 1 Gratings lens parameters } \\
\hline$E_{\text {bog }}$ & GeV & 1 & 1000 \\
\hline$E_{S}$ & $G V / m$ & 1 & 1 \\
\hline$B_{S}$ & $T$ & 3.3 & 3.3 \\
\hline$L_{0}$ & $\mathrm{n}$ & 1 & 1000 \\
\hline$\lambda$ & $\mu m$ & 10.6 & 10.6 \\
\hline$\phi$ & & $60^{\circ}$ & $60^{\circ}$ \\
\hline$\Phi$ & & $0^{\circ}$ & $0^{\circ}$ \\
\hline$G$ & $T / m$ & $5 \times 10^{5}$ & $5 \times 10^{5}$ \\
\hline$f$ & $\mathrm{~m}$ & $7 \times 10^{-4}$ & 0.7 \\
\hline
\end{tabular}

The effective gradient for a $1 \mathrm{GV} / \mathrm{m}$ surface field exceeds $10^{5} \mathrm{~T} / \mathrm{m}$. The focal length of the lens is less than $1 \mathrm{~mm}$ for a $1 \mathrm{GeV}$ beam.

\section{Survey of the theoretical literature}

We describe in this section calculations concerning the acceleration of particles using a grating structure that have appeared in the literature. We restrict ourselves here to papers dealing with true gratings.

The first proposal to use a laser as the power source for an electron accelerator was made by Shimoda[14] in 1962. Although he discussed phase matching using dielectrics and the inverse Cerenkov effect, he did not consider the possibility of using a grating.

The first person to consider using an illuminated grating as an accelerator seems to be Lohmann[15]. He apparently made the connection between acceleration over a grating and the SmithPurcell effect.

Takeda and Matsui[16] proposed using a grating inside a laser cavity as an electron accelerator. The metallic grating also acted as one "mirror" in the laser resonator cavity. The radiation beam reflects along the $y$ axis.

Mizuno, Ono, and Shimoe[17] pointed out that grating acceleration and the Smith-Purcell effect are related through time reversal of the electron and radiation directions. Both require a synchronism condition between the electron velocity and the phase velocity of the laser. They propose a configuration with the grating acting as one mirror in the laser resonator, but the angle of the incident 
light is allowed to vary in the $y-z$ plane.

The idea of accelerating electrons up to high energies with gratings was given an important boost in 1980 in a seminal paper by Palmer[5]. He pointed out that phase synchronism with a relativistic electron was possible, provided the electrons do not travel over the grating with their velocity perpendicular to the grating grooves, or if the incident radiation does not lie in the $y-z$ plane. He seems to be the first to propose an accelerator geometry where the lasers are treated as power sources independent of the gratings, which are treated as accelerator cavities. He gives a treatment of the fields above the grating using an expansion of evanescent space harmonics. He proposed a specific grating profile that would support a $\pi$ accelerating mode with an accelerating field enhancement of 5 .

In one of the first attempts to consider the more "practical" considerations, Palmer found that surface losses would limit the achievable field enhancement to $\approx 5$. He considered the maximum power densities at which the grating might survive. He showed that the total laser energy required per meter of accelerator is quite small, mainly due to the small cavity volume. He argued that the phase acceptance, and the vertical and horizontal stability are acceptable.

Palmer has elaborated on the discussion of these practical considerations[18]. A parameter list was given for a hypothetical $50 \mathrm{TeV}$ linear collider.

Laser acceleration of electrons using gratings was extensively discussed at a workshop[19] at Los Alamos in 1982. The status of the overall concept was reviewed by a working group headed by Palmer[20]. The group compared many of the properties of conventional accelerator cavities with those of a grating linac. They concluded that grating structures have a shunt impedance a factor of 5 lower and thus are intrinsically less efficient. They derived scaling relations showing how the power loss, RF energy, cavity $Q$, and fill time vary wi,h wavelength. They showed that the fraction of the stored $R F$ power that could be given to the particle beam (loading) is independent of wavelength. They examined the limits on the achievable accelerating field due to electrical breakdown, heating, and plasma formation.

Kim and Kroll made a detailed study of the requirements for tranverse stability in a grating linac[12]. They showed that the forces on a particle above the grating are quadrupole-like, focusing in one dimension and defocussing in the other. They proposed achieving stability by periodically interupting the grating shape, thereby altering the RF phase seen by the particles. This requirement puts a constraint on the stable region of phase acceptance. 
Kroll studied[21] the sensitivity of the grating linac to grating errors. He found that deviations of the particles from the nominal trajectory arising from random groove displacements was of the order of the groove errors themselves.

Channel examined[22] the question of surface damage on gratings. He showed that a dense plasma may form over the grating surface when it is used with incident field strengths near the damage threshold.

The connection between the true grating structures discussed in this report and the more general class of open accelerating structures has been given[23-4] by Palmer.

The use of a rectangular grooved grating as an accelerator was studied in great detail by Pickup[11,25]. He developed a code that determined the fields above the grating by matching a sum in space harmonics above the grooves with a modal expansion within the grooves. Using the code he was able to predict the resonance frequency, shunt impedance, and cavity $Q$ of the standing wave modes expected in grating structures. He showed that the efficiency (R/Q) of the grating scaled to $2856 \mathrm{MHz}$ was about $\frac{1}{2}$ that of a SLAC cavity. The group velocity was about 10 times higher. He simulated the single particle motion down an accelerator. Strong focusing was achieved by periodically rotating short sections of grating by $90^{\circ}$ around the beam axis. He derived emittance and phase acceptance limits for the captured particles. He showed that the expected energy loss due to synchrotron radiation was small compared with the expected energy gain for a $1 \mathrm{TeV}$ electron in a laser linac. He calculated the expected wakefields in the structure by numerically integrating Maxwell's equations in the time domain. At $2856 \mathrm{MHz} a$ single bunch transverse instability limited the maximum charge per bunch to about 3 times that for a SLAC cavity.

Bae, Furuya, Shirai, Nozokido and Mizuno[26] designed a $\pi$ mode grating suitable for acceleration studies using 496 um radiation. They optimized the width and depth of the rectangular grooves, taking into account the effects of the finite resistivity of the grating material.

There have been a number of reviews of new acceleration techniques that also consider grating acceleration[27-32].

\section{Survey of the experimental literature}

We mention here the small amount of experimental work directly related to accelerating particles using the fields over true gratings. In addition to this we should add that all the experimental observations of Smith-Purcell radiation provide an indirect proof that this acceleration method must work at some level. 
Takeda and Matsui[16] proposed using a grating inside a laser cavity as an electron accelerator. The metallic grating also acts as one "mirror" in the laser resonator cavity. They used a $Q-$ switched ruby laser with a peak power of $1 \mathrm{MW} / \mathrm{em}^{2}$ and $50 \mathrm{~ns}$ pulse length. The radiation had H-polarization and was incident normal to the grating surface $\left(\phi=90^{\circ}\right)$. The echelette-shaped grating used an aluminum layer deposited on glass. They found that the laser power damaged the grating.

Pickup [11,25] made microwave measurements of a model of a grating accelerator. He used a rectangular grooved copper grating $w i t h D=$ $1.52 \mathrm{~cm}$ and $\mathrm{H}=0.52 \mathrm{~cm}$ and a total of 16 grooves. He formed $a$ cavity open on the top by adding end and side walls. The grating profile was chosen to support a $\pi$ accelerating mode $(k D=\pi)$ for relativistic particles. The location of the side walls restricts the possible wavevectors along the $x$ direction.

Coaxial probes centered in the end walls were used to make microwave transmission measurements. This arrangement was only capable of exciting longitudinal electric (H-polarization) modes. He measured $a Q$ value of 9800 at a resonant frequency of $9.89 \mathrm{GHz}$. The shunt impedance was $2.2 \times 10^{4} \Omega / \mathrm{cm}$. Besides the $\pi$ mode a number of other modes down to $\pi / 2$ were observed, all of which agreed well with calculations.

The first experimental observation of electron acceleration over a grating was made by Mizuno, Pae, Nozokido, and Furuyal33]. The rectangular-grooved grating was excited using a $\mathrm{CH}_{3} \mathrm{~F}$ laser at a wavelength of $496 \mathrm{\mu m}$. The laser produced a peak power of $2.5 \mathrm{~W}$ with a $100 \mathrm{~ns}$ pulse length. The incident beam came from a $80 \mathrm{kV}$ electron gun. The interaction length was $2 \mathrm{~mm}$. The energy of the electrons after passing over the grating was analyzed using a retarding field detector with a resolution of $0.8 \mathrm{eV}$. The detector current as a function of the grid bias clearly showed the presence of accelerated and decelerated electrons. The signal went away when the laser pulse was not present. The FWHM of the energy spread introduced by the laser was around $5 \mathrm{eV}$.

In a second measurement[34] the laser power was raised to $4.5 \mathrm{~W}$ and the measured FWHM of the energy spread increased to $\approx 20 \mathrm{eV}$. In a third measurement [35] the laser power was raised to $30 \mathrm{~W}$ and the measured FWHM of the energy spread was $100 \mathrm{eV}$. The incident laser beam came down along the normal to the grating surface. The energy spread was found to grow quadratically with the peak power of the incident laser light, as expected. The energy spread over a given grating was found to peak for a certain value of the incident beam energy, as expected. The interaction length was determined to be 3 $\mathrm{mm}$. 


\section{Conclusions}

We have seen that it should be possible to use grating structures to accelerate relativistic particles. Efficient surfaces can be designed with large accelerating field enhancements. These structures are not restricted to use at optical wavelengths. Although they can be scaled for use at any wavelength, it seems likely that any practical applications will occur in the wavelength regime between $10 \mu \mathrm{m}$ and $1 \mathrm{~mm}$, where building a conventional closed cavity would be very difficult or impossible.

The problems with the vertical force and the lack of a neutral axis probably rules out using true gratings as the accelerator sections in linear colliders. These problems have lead to the study of more general "open structures" that would be more suitable for this type of application\{23\}. Certain of these grating-like structures could make an acceptable accelerating structure. However, it is now believed that any acceleration technique at optical frequencies will be unsuitable for linear colliders[36]. Thus, if the grating structure is to play any role as an accelerator, it will probably be used in conjunction with an infrared or microwave power source.

A grating structure may useful for other specialized applications. We have mentioned the possibility of using a grating as a high power lens. Other possible applications include a buncher, deflector, room sized GeV electron accelerator, or free electron laser. 


\section{Endnotes and references}

[1] A. Sessler, The quest for uitrahigh energies, Am. J. Phys. $54: 505-10,1986$.

[2] R. Petit (ed), Eiectromagnetic Theory of Gratings, SpringerVerlag, 1980 .

[3] J.W.S. Rayleigh, On the dynamical theory of gratings, Proc. Roy. Soc. A 79:399-4l6, 1907 .

[4] P.M. Van den Berg, Diffraction theory of a reflection grating, Appl. Sci. Res. 24:261-93, 1971.

[5] R.B. Palmer, A laser drivengrating 1inac, Part. Acc. 11:81-90, 1980 .

[6] S. Smith \& E. Purcell, Visible light from localized surface charges moving across a grating, Phys. Rev. 92:1069, 1953.

[7] S. Smith, Visible light from localized surface charges moving across a grating, Thesis, Harvard University, 1953.

[8] P. van den Berg, Smith-Purcell radiation from a point charge moving parallel to a reflection grating, J. Opt. Soc. Am. 63:158897,1973 .

[9] The GERTY code is an implementation of the integral equation method given by van den Berg in reference 4 above.

[10] Additional results concerning optimization of sinusoidal surface boundaries can be found in R. Fernow, Effects of SmithPurcell radiation on the laser acceleration experiment, unpublished report RCF-LA-8701, 1987 .

[11] M. Pickup, A grating linac at microwave frequencies, in $C$. Joshi \& T. Katsouleas(eds), Laser Acceleration of Particles, $p$. 281-95, AIP Conf. Proc. No. 130, 1985.

[12] K. Kim \& N. Kroll, Some effects of the transverse stability requirement on the design of a grating linac, in P. Channelledl, Laser Acceleration of Particles, AIP Conf. Proc. 91, p.190-8, 1982. [13] R. Fernow, Design of a grating for studying Smith-Purcell radiation and electron acceleration, in C. Joshi(ed), Advanced Accelerator Concepts, AIP Conf. Proc. 193, P.87-103, 1989 .

[14] K. Shimoda, Proposal for an electron accelerator using an optical maser, Applied Optics $1: 33-5,1962$.

[15] A. Lohmann, Electron acceleration by light waves, unpublished IBM technical note TN5, 1962. This work was pointed out by Lawson in reference 28 below.

[16] Y. Takeda \& I. Matsui, Laser 1inac. with grating, Nuc. Instr. Meth. 62:306-10, 1968 .

[17] K. Mizuno, S. Ono \& 0 . Shimoe, Interactions between coherent light waves and free electrons with a reflection grating, Nature $253: 184-5,1975$.

[18] R. Palmer, A laser driven grating linac, in J. Mulvey(ed), The challenge of ultra-high energies, ECFA report $83 / 68,1982$.

[19] P. Channel(ed), Laser Acceleration of Particles, AIP Conf. Proc. 91,1982 . 
[20] R. Palmer, Near field accelerators, in P. Channel(ed), op cit, p. $179-89$.

[21] N. Kroll, Sensitivity of a laser driven grating linac to grating errors, in P. Channel(ed), op cit, p.237-43.

[22] P. Channel, Are gratings invisible?, in P. Channel(ed), op cit, p.244-7.

[23] R. Palmer, Open accelerating structures, in S. Turner(ed), New Developments in Particle Acceleration Techniques, CERN report 8711, p. $633-41,1987$.

[24] R. Palmer et al, Report of the near field group, in C. Joshi \& T. Katsouleas(eds), Lager Acceleration of Particles, AIP Conf. Proc. 130, p.234-52, 1885 .

[25] M. Pickup, A linear accelerator using gratings, Thesis, Cornell University, 1987.

[28] J. Bae, K. Furuya, H. Shirai, T. Nozokido \& K. Mizuno, The inverse Smith-Purcell effect in the submillimeter wave regiontheoretical analyses, Jap. J. Appl. Phys. 27:408-12, 1988.

$\lfloor 27\rfloor$ R. Pantell, Interactions between electromagnetic fields and electrons, in R. Carrigan, F. Huson, \& M. Month(eds), Physics of High Energy Particle Accelerators, AIP Conf. Proc. 87, p.863-918, 1981 .

[28] J. Lawson, Laser accelerators: where do we stand?, in $P$. Bryant \& J. Mulvey(eds), The generation of high fields for particle acceleration to very high energies, CERN report 85-07, p.3-12, 1985 .

[29] J. Lawson \& M. Tigner, The physics of particle accelerators, Ann. Rev. Nucl. Part. Sci. 34:99-123, 1984.

[30] $R$. Palmer \& $R$. Fernow, New acceleration methods for high energy physics, in M. Month, P. Dahl \& M. Dienes(eds), Physics of High Energy Particle Accelerators, AIP Conf. Proc. 127, p.948-63. 1.985 .

[31] J. Arnesson \& F. Kneubuhl, Future laser driven particle accelerators, Infrared Phys. 25:121-31, 1985 .

[32] R. Palmer, An introduction to acceleration mechanisms, in Frontiers of Particle Beams, Springer-Verlag Lecture Notes in Physics 296, p.607, 1986 .

[33] K. Mizuno, J. Pae, T. Nozokido \& K. Furuya, Experimenta] evidence of the inverse Smith-Purcell effect, Nature 328:45-7, 1987 .

[34] J. Pae, K. Furuya, H. Shirai, T. Nozokido \& K. Mizuno, First observation of the inverse Smith-Purcell effect, IEEE IEDM 87:307310,1987 .

[35] J. Bae, K. Furuya, H. Shirai, T. Nishida, T. Nozokido \& K. Mizuno, The inverse Smith-Purcell effect at submillimeter wavelengths, F3.4, p. 412-3, reference unknown.

[36] R. Palmer, Prospects for high energy $e^{t} e^{*}$ linear colliders, Ann. Rev. Nucl. Part. Sci. 40:529-92, 1990. 